\title{
The ISO view of Palomar-Green quasars ${ }^{\star}$
}

\author{
M. Haas ${ }^{1}$, U. Klaas ${ }^{1}$, S. A. H. Müller ${ }^{2}$, F. Bertoldi ${ }^{3}$, M. Camenzind ${ }^{4}$, R. Chini ${ }^{2}$, O. Krause ${ }^{1}$, D. Lemke ${ }^{1}$, \\ K. Meisenheimer ${ }^{1}$, P. J. Richards ${ }^{5}$, and B. J. Wilkes ${ }^{6}$ \\ 1 Max-Planck-Institut für Astronomie (MPIA), Königstuhl 17, 69117 Heidelberg, Germany \\ 2 Astronomisches Institut, Ruhr-Universität Bochum, 44780 Bochum, Germany \\ 3 Max-Planck-Institut für Radioastronomie, Auf dem Hügel 69, 53121 Bonn, Germany \\ ${ }^{4}$ Landessternwarte Heidelberg, Königstuhl, 69117 Heidelberg, Germany \\ 5 CLRC, Rutherford Appleton Laboratory, Chilton, Didcot, OX11 0QX, UK \\ ${ }^{6}$ Harvard-Smithsonian Center for Astrophysics, 60 Garden Street, Cambridge MA 02138, USA
}

Received 2 September 2002 / Accepted 24 January 2003

\begin{abstract}
Mining the ISO data archive we provide the complete ISO view of PG quasars containing 64 infrared spectral energy distributions between 5 and $200 \mu \mathrm{m}$. About half of the sample was supplemented by MAMBO and SCUBA (sub-)millimetre data. Since the PG quasars were selected optically, the high infrared detection rate of more than $80 \%$ suggests that every quasar possesses luminous to hyperluminous dust emission with dust masses comparable to Seyferts and ultraluminous IR galaxies (ULIRGs). The gas-to-dust mass ratio (of those sources where CO measurements are available in the literature) is consistent with the galactic value providing further evidence for the thermal nature of the IR emission of radio quiet quasars. The SEDs represent templates of unprecedented detail and sensitivity. The power-law like near- to mid-IR SEDs $\left(F_{v} \propto v^{\alpha}\right)$ are smooth up to far-infrared wavelengths, favouring dust heating by the central AGN, and we conclude that, in particular for our hyperluminous quasars at $z=1$, starbursts play only a minor role for powering the dust emission, even in the FIR. The IR spectral slopes $\alpha_{1-10 \mu \mathrm{m}}$ range from -0.9 to -2.2 with a mean of $-1.3 \pm 0.3$. They neither correlate with the optical spectral slope $\alpha_{0.3-1 \mu \mathrm{m}}$, nor with the IR luminosity, nor with the FIR/MIR luminosity ratio, nor with inclination-dependent extinction effects in the picture of a dusty torus. We suggest that the diversity of the SEDs reflects largely the evolution of the dust distribution, and we propose a classification of the SED shapes as well as an evolutionary scheme in which this variety can be understood. During the evolution the surrounding dust redistributes, settling more and more into a torus/disk like configuration, while the SEDs show an initial FIR bump, then an increasing MIR emission and a steeper near- to mid-infrared slope, both of which finally also decrease. Strikingly, based on the sensitive ISO data now we do not only see the coarse IR differences between ULIRGs and quasars, but also the details and a possible evolution of the dust distribution and emission even among the optically selected PG sample. Regarding cosmic evolution, our hyperluminous quasars in the "local" universe at $z=1$ do not show the hyperluminous $\left(L_{\mathrm{FIR}} \gtrsim 10^{13} L_{\odot}\right)$ starburst activity inferred for $z=4$ quasars detected in several (sub-)millimetre surveys. In view of several caveats this difference should be established further, but it already suggests that in the early dense universe stronger merger events led to more powerful starbursts accompanying the quasar phenomenon, while at later cosmic epochs any coeval starbursts obviously do not reach that high power and are outshone by the AGN.
\end{abstract}

Key words. galaxies: fundamental parameters - galaxies: photometry - galaxies: quasars: general - infrared: galaxies

\section{Introduction}

The Palomar-Green catalog (Schmidt \& Green 1983) provides an optically selected sample of 114 quasars, about $30 \%$ of which were detected by IRAS in the representative midinfrared $25 \mu \mathrm{m}$ and far-infrared $60 \mu \mathrm{m}$ bands (cf. Neugebauer et al. 1986; Sanders et al. 1989). Sanders et al. (1988a,b) proposed that quasars are preceeded by a dusty ULIRG phase. Barvainis (1990) and Elvis et al. (1994) emphasized the

Send offprint requests to: M. Haas, e-mail: haas@mpia.de

* Based on observations with the Infrared Space Observatory ISO, an ESA project with instruments funded by ESA Member States (especially the PI countries: France, Germany, The Netherlands and the UK) and with the participation of ISAS and NASA. dispersion of IR SEDs. Rowan-Robinson (1995) proposed that, in addition to the AGN which heats the NIR and MIR emission, starbursts are required to power the FIR emission. On the basis of a few ISO observations Haas et al. (1998) demonstrated that the IR emission of quasars is a mixture of thermal and synchrotron radiation, dominated by thermal emission in radio-quiet quasars and synchrotron emission in flat spectrum radio-loud ones.

In Haas et al. (2000, henceforth Paper I) we reported on 17 PG quasars observed by ISO and the IRAM 30-m telescope as part of the ISOPHOT guaranteed time. The detection rate was about $70 \%$, providing evidence for luminous to hyperluminous dust emission in these optically selected sources. The variety of SED shapes and the correlation between mid-and 
far-infrared luminosities was discussed in light of a possible AGN-starburst connection. The data support the picture that the MIR emission is mainly powered by the AGN, while - with respect to the available AGN dust torus models - starbursts may be required to provide the high FIR luminosity. There is a marginal trend that the hyperluminous quasars have a higher MIR/FIR luminosity ratio, indicating differences between them and the low luminosity quasars.

In order to put the results drawn from the small sample of 17 sources onto a broader statistical basis, we here present the mostly unpublished SEDs of the remaining 47 PG quasars in the ISO archive. We discuss the statistical properties of the complete data base of all 64 PG quasars observed by ISO and propose an evolutionary sequence to interpret the diversity of the infrared properties. Since no selection criteria except visibility to the satellite were applied, this sample provides a representative selection from the 114 quasars in the PG cata$\log$, with a redshift range from nearby to distant $(z \lesssim 2)$ objects. Throughout this paper we use $H_{0}=75 \mathrm{~km} \mathrm{~s}^{-1} \mathrm{Mpc}^{-1}$ and $q_{0}=0$, and blackbodies modified with a dust emissivity index of $\beta=2$.

\section{The data}

The ISO Data Archive contains photometric observations with ISOPHOT and ISOCAM for 64 PG quasars. For some of these we found complementary submm photometry in the JCMTSCUBA archive, and performed additional mm observations with the Max-Planck millimeter bolometer array MAMBO at the IRAM 30-m telescope.

\subsection{ISOPHOT and ISOCAM}

The ISOPHOT data of the new 47 PG quasars were observed and evaluated along the same lines as those 17 sources described in Paper I.

The observations were performed with ISOPHOT (Lemke et al. 1996), the photometer on board ISO (Kessler et al. 1996). The observing modes (Laureijs et al. 2002) comprise chopped measurements (including those with the spectrometer ISOPHOT-S) and small maps.

The data were reduced using the ISOPHOT Interactive Analysis tool (PIA ${ }^{1}$ V9.1 and V10), together with the calibration data set V7.0. Using the latest versions of the data reduction tools, there are no systematic photometric offsets with regard to PIA V7.0 used for the 17 sources in Paper I. The accuracy of the absolute photometric calibration depends mainly on systematic errors as described in Laureijs \& Klaas (1999), and it is currently known to be better than $30 \%$ for faint sources, and the relative filter-to-filter calibration is better than $15 \%$ (Klaas et al. 2002).

Some sources were observed in the MIR at 6.7, 9.6, 12 and $14.3 \mu \mathrm{m}$ with ISOCAM (Cesarsky et al. 1996). The images were retrieved from the ISO data archive (Kessler et al. 2000) and cleaned from residual glitches. Photometry was

\footnotetext{
${ }^{1}$ PIA is a joint development by the ESA Astrophysics Division and the ISOPHOT consortium led by the MPI für Astronomie, Heidelberg. Contributing institutes are DIAS, RAL, AIP, MPIK, and MPIA.
}

derived using $21^{\prime \prime}$ square apertures. The photometric accuracy is currently known to be better than $30 \%$ (Cesarsky \& Blommaert 2001).

In addition, for 17 of the 64 PG quasars the ISO Data archive contains MIR spectra, 12 taken with ISOPHOT-S $(6-12 \mu \mathrm{m})$ and 7 with ISOCAM-CVF $(10-16 \mu \mathrm{m})$. At the faint flux level the uncertainty is about $30-50 \%$. Therefore the spectral features are considered with some reservation, but the continuum estimates are consistent with the photometic data, and upper limits for the PAH features are derived.

\subsection{JCMT-SCUBA and IRAM-MAMBO}

Yet unpublished submm continuum data obtained with SCUBA in 1998 were retrieved from the JCMT archive. The observations were performed in photometry mode and reduced with the SCUBA User Reduction Facility (SURF) including identification of noisy bolometer pixels and removal of sky noise. The atmospheric transmission was determined from skydips and water radiometer data at the CSO. IRC +10206 and HL Tau served as standard calibrators. The integration time per source was $12-15 \mathrm{~min}$. Due to very good atmospheric transmission $\left(\tau_{\mathrm{CSO}}=0.016\right)$ during the observation of PG1440+356, we also included $450 \mu \mathrm{m}$ data for this source. The photometric accuracy at $450 \mu \mathrm{m}$ and $850 \mu \mathrm{m}$ is around $30 \%$.

The 1.2-mm continuum observations were carried out with the Max-Planck millimeter bolometer array MAMBO (Kreysa et al. 1998) at the IRAM 30-m telescope on Pico Veleta during dedicated backup time between 2002 March 22 and May 21. All objects were observed at least twice, on different days. The integration time per source varied between 2.5 and $12 \mathrm{~min}$. The data were reduced using the MOPSI software package. The atmospheric transmission was intermediate with $\tau(1.2 \mathrm{~mm})$ between 0.15 and 0.5 ; the absolute calibration was established by observations of Mars and Uranus. The uncertainty of the 1.2-mm fluxes is estimated to be around $20 \%$.

\section{Results}

The IR and mm fluxes are listed in Table 1 (where we do not repeat the values of the 17 sources of Paper I). ISO achieved about $95 \%$ and $80 \%$ detections in the MIR $(\leq 25 \mu \mathrm{m})$ and FIR $(\geq 60 \mu \mathrm{m})$, respectively. Even at the long wavelengths between 120 and $200 \mu \mathrm{m}$ the detection rate is about $30 \%$. The fluxes of the five brightest sources detected also by IRAS (PG0157+001, PG1351+640, PG1440+356, PG1700+518, PG2130+099) agree within 15\%. 12 sources listed as marginal detections in the IRAS Faint Source catalog are now clearly confirmed by ISO, and 30 new detections were achieved. Note that three sources (PG1001+054, PG1211+143, PG1351+640) are located in cirrus regions, such that the chopped observations failed in the FIR, in particular longwards of $100 \mu \mathrm{m}$, but with the help of the maps the quasar could be discerned from the local foreground. Some discrepancies between our results and those listed in Sanders et al. (1989) find a natural explanation by the cirrus which even for the IRAS pointed observations could not be resolved sufficiently due to the large IRAS beam of about $3^{\prime}$. 
Table 1. Measured flux densities in mJy as a function of wavelength in $\mu \mathrm{m}$. Bold numbers are detections above the 3- $\sigma$ level, the errors are $10-30 \%$. The thin numbers represent 3- $\sigma$ upper limits. The apertures typically used for each filter are listed below the wavelength; for $120-200 \mu \mathrm{m}$ the apertures were $90^{\prime \prime}$ in the case of maps and $180^{\prime \prime}$ in the case of chopped photometry, the latter case always leading to upper limits only.

\begin{tabular}{|c|c|c|c|c|c|c|c|c|c|c|c|c|c|c|c|c|c|c|c|c|c|c|}
\hline$\overline{\mathrm{PG}}$ & $\begin{array}{c}3.6 \\
5^{\prime \prime}\end{array}$ & $\begin{array}{r}4.8 \\
23^{\prime \prime}\end{array}$ & $\begin{array}{c}6.7^{*} \\
21^{\prime \prime}\end{array}$ & $\begin{array}{r}7.3 \\
52^{\prime \prime}\end{array}$ & $\begin{array}{r}9.6 \\
21^{\prime \prime}\end{array}$ & $\begin{array}{l}12.0 \\
52^{\prime \prime}\end{array}$ & $\begin{array}{l}14.3^{*} \\
21^{\prime \prime}\end{array}$ & $\begin{array}{r}16 \\
52^{\prime \prime}\end{array}$ & $\begin{array}{r}20 \\
52^{\prime \prime}\end{array}$ & $\begin{array}{r}25 \\
52^{\prime \prime}\end{array}$ & $\begin{array}{r}60 \\
45^{\prime \prime}\end{array}$ & $\begin{array}{r}80 \\
45^{\prime \prime}\end{array}$ & $\begin{array}{r}90 \\
45^{\prime \prime}\end{array}$ & $\begin{array}{l}100 \\
45^{\prime \prime}\end{array}$ & 120 & 150 & 170 & 180 & 200 & $\begin{array}{r}450 \\
8^{\prime \prime}\end{array}$ & $\begin{array}{l}850 \\
15^{\prime \prime}\end{array}$ & $\begin{array}{r}1200 \\
11^{\prime \prime}\end{array}$ \\
\hline$\overline{0003+199}$ & & & 141 & & 179* & & & 351 & & 391 & & & & & & & & & & & & \\
\hline $0007+106$ & & & & & & $62^{*}$ & & & & & 171 & & & 221 & & $<288$ & & & 271 & & & \\
\hline $0157+001$ & & 27 & & 45 & & 134 & & & & 570 & 2210 & & & 2000 & & 1050 & & & 650 & & & \\
\hline $0804+761^{a}$ & & 51 & & 76 & & 130 & & & & 218 & 188 & & & 121 & & $<60$ & & & $<48$ & & & $<5.4^{g}$ \\
\hline $0838+770$ & & & & & & & & & & 85 & 167 & & & 180 & & 150 & & & 92 & & & \\
\hline $1001+054$ & & 16 & & 29 & & 63 & & & & 109 & 140 & & & 146 & & $<285$ & & & $<255$ & & & \\
\hline $1008+133$ & & 5 & & 12 & & 34 & & & & 108 & $<132$ & & & $<246$ & & $<462$ & & & $<660$ & & & $<6.9$ \\
\hline $1100+772^{b}$ & & 9 & & 14 & & 18 & & & & 40 & 60 & & & 40 & & $<90$ & & & $<90$ & & & \\
\hline $1114+445$ & & 26 & & 47 & & 87 & & & & 146 & $<237$ & & & 200 & & $<300$ & & & $<324$ & & & $<9.3$ \\
\hline $1116+215$ & & 44 & & 73 & & 152 & & & & 187 & $<219$ & & & $<285$ & & $<351$ & & & $<396$ & & & $<9.9$ \\
\hline $1149-110$ & & 20 & & 46 & & 120 & & & & 272 & 368 & & & 314 & & $<390$ & & & $<627$ & & & $<5.1$ \\
\hline $1211+143$ & & 60 & & 100 & & 160 & & & & 300 & 518 & 392 & & $<279$ & & $<309$ & $<306$ & & $<408$ & & & \\
\hline $1216+069^{b}$ & & 16 & & 28 & & 62 & & & & 95 & $<132$ & & & $<129$ & & $<288$ & & & $<705$ & & & 7.3 \\
\hline $1226+023^{c}$ & & & 194 & & & & 294 & & & & 1124 & 1291 & & 1348 & 1546 & 1113 & 1292 & 1056 & 1091 & & & \\
\hline $1229+204$ & & 26 & & 38 & & $<69$ & & & & 158 & 241 & & & 317 & & $<288$ & & & $<435$ & & & \\
\hline $1244+026$ & & 15 & & 37 & & 86 & & & & 206 & 368 & & & 362 & & $<279$ & & & $<633$ & & & $<4.6$ \\
\hline $1248+401$ & & & & & & & & & $<45$ & & & & & & & & & & & & & \\
\hline $1309+355$ & & 18 & & 30 & & 60 & & & & 102 & $<162$ & & & $<192$ & & $<207$ & & & $<261$ & & & $<8.1$ \\
\hline $1322+659$ & & & & 18 & & 34 & & & & 54 & 90 & & & 100 & & $<93$ & & & $<75$ & & & $<5.3$ \\
\hline $1338+416$ & & & & & $10^{s}$ & & & & 41 & & & & & & & & & & & & & \\
\hline $1351+640$ & & $<36$ & & 55 & & 135 & & & & 550 & 719 & & & 526 & & & & & & & & \\
\hline $1352+183^{b}$ & & & & & & & & & & & 197 & 187 & & $<180$ & & & & & & & & \\
\hline $1354+213^{b}$ & & & & 8 & & 17 & & & & 34 & $<264$ & $<186$ & & $<165$ & & $<150$ & & & $<204$ & & & \\
\hline $1402+261$ & & & & 38 & & 69 & & & & 120 & 209 & & & 213 & & 139 & & & $<132$ & & $<7.2$ & $<6.6$ \\
\hline $1404+226^{d}$ & & & & 11 & & 26 & & & & 62 & $<144$ & 152 & & 123 & & $<114$ & $<114$ & & $<126$ & & & \\
\hline $1407+265$ & & $<9$ & & 17 & & & & & & 78 & 171 & & & $<200$ & & $<507$ & & & $<528$ & & & $<6.6$ \\
\hline $1415+451$ & & & & 27 & & 44 & & & & 71 & 110 & & & 147 & & 136 & & & 92 & & & $<5.7$ \\
\hline $1416-129$ & & 13 & & 25 & & 55 & & & & 105 & $<129$ & & & $<132$ & & $<351$ & & & $<741$ & & & $<8.1$ \\
\hline $1425+267$ & & & & & & $19 *$ & & & & & & & & & & & & & & & & $<4.8$ \\
\hline $1426+015$ & & 49 & & 81 & & 136 & & & & 271 & & 350 & & 350 & & 312 & & & 202 & & & \\
\hline $1427+480$ & & & & 10 & & 17 & & & & 48 & 82 & & & 92 & & 61 & & & $<60$ & & & $<5.7$ \\
\hline $1435-067^{b}$ & & & & 20 & & 50 & & & & $<126$ & 304 & $<504$ & & $<333$ & & $<387$ & $<369$ & & $<318$ & & & \\
\hline $1440+356^{a}$ & & 40 & & 54 & & 127 & & & & 211 & 597 & & & 780 & & 627 & & & 314 & $<37.7$ & $<5.2$ & \\
\hline $1444+407$ & & & & 21 & & 59 & & & & 107 & 125 & & & 80 & & $<60$ & & & $<60$ & & & $<5.1$ \\
\hline $1501+106$ & & & 69 & & $122^{*}$ & & & 277 & & 464 & 750 & & 635 & & & & & & & & & \\
\hline $1512+370$ & & 9 & & $<18$ & & 36 & & & & 50 & 65 & & & $<84$ & & $<120$ & & & $<96$ & & $<8.3$ & $<6.0$ \\
\hline $1519+226^{b}$ & & & & 40 & & 59 & & & & 80 & $<141$ & $<183$ & & $<195$ & & $<480$ & $<360$ & & $<1080$ & & & \\
\hline $1543+489^{b}$ & & 12 & & 23 & & 50 & & & & 126 & 300 & & & 280 & & 150 & & & $<189$ & & & \\
\hline $1545+210^{e}$ & & 11 & & 16 & & 25 & & & & 43 & 45 & & 33 & & & & $<57$ & & & & & \\
\hline $1626+554$ & & 9 & & 20 & & 38 & & & & 50 & $<156$ & & & 70 & & $<150$ & & & $<123$ & & & $<3.7$ \\
\hline $1630+377^{f}$ & & & 5 & & & & 9 & & & & & & & & & & $<30$ & & & & & \\
\hline $1700+518$ & & 31 & & 50 & & 96 & & & & 250 & 348 & & & 374 & & 277 & & & 142 & & $<10.8$ & \\
\hline $1704+608^{e}$ & & 18 & & 31 & & 50 & & & & 112 & 201 & & 168 & 151 & & 103 & 73 & & 43 & & & \\
\hline $1715+535^{f}$ & & & 6 & & & & 12 & & & & & & & & & $<42$ & & & & & & \\
\hline $1718+481^{b}$ & & 11 & & 19 & & 30 & & & 45 & 52 & 72 & & & 58 & & $<63$ & & & $<81$ & & & $32.0^{g}$ \\
\hline $2130+099$ & & 75 & & 114 & & 185 & & & & 333 & 480 & & & 485 & & 384 & & & 242 & & & \\
\hline $2308+098^{b}$ & $<9$ & $<12$ & & $<15$ & & 20 & & & & 56 & $<180$ & 180 & & $<165$ & & $<570$ & $<531$ & & $<1095$ & & & 8.5 \\
\hline
\end{tabular}

* With ISOCAM.

${ }^{a}$ See also Wilkes et al. (2000).

${ }^{b}$ Consistent with Polletta et al. (2000), see also Wilkes et al. (1999).

${ }^{c}$ PG $1226+023=3$ C 273, FIR values taken from Meisenheimer et al. (2001).

${ }^{d}$ Consistent with Polletta \& Courvoisier (1999).

${ }^{e}$ PG 1545+210 = 3C 323.1, PG 1704+608 = 3C 351, see also van Bemmel et al. (2000).

${ }^{f}$ Consistent with Oyabu et al. (2001).

${ }^{g}$ At $1300 \mu \mathrm{m}$.

${ }^{s}$ Continuum at $9.6 \mu \mathrm{m}$ from ISOPHOT-S.

Twenty sources were observed with MAMBO at $1.2 \mathrm{~mm}$, of which three were detected, and 3- $\sigma$ upper flux limits are provided for the others. Upper $850 \mu \mathrm{m}$ flux limits were derived for four sources from data in the SCUBA archive (and one of them also at $450 \mu \mathrm{m})$.
Supplementing our data with further measurements found in the literature, in Fig. 1 we display the observed (uncorrected for redshift!) spectral energy distributions (SEDs) of the 47 quasars. Note that the SED curves run smoothly and the photometric uncertainties represented by the size of the 


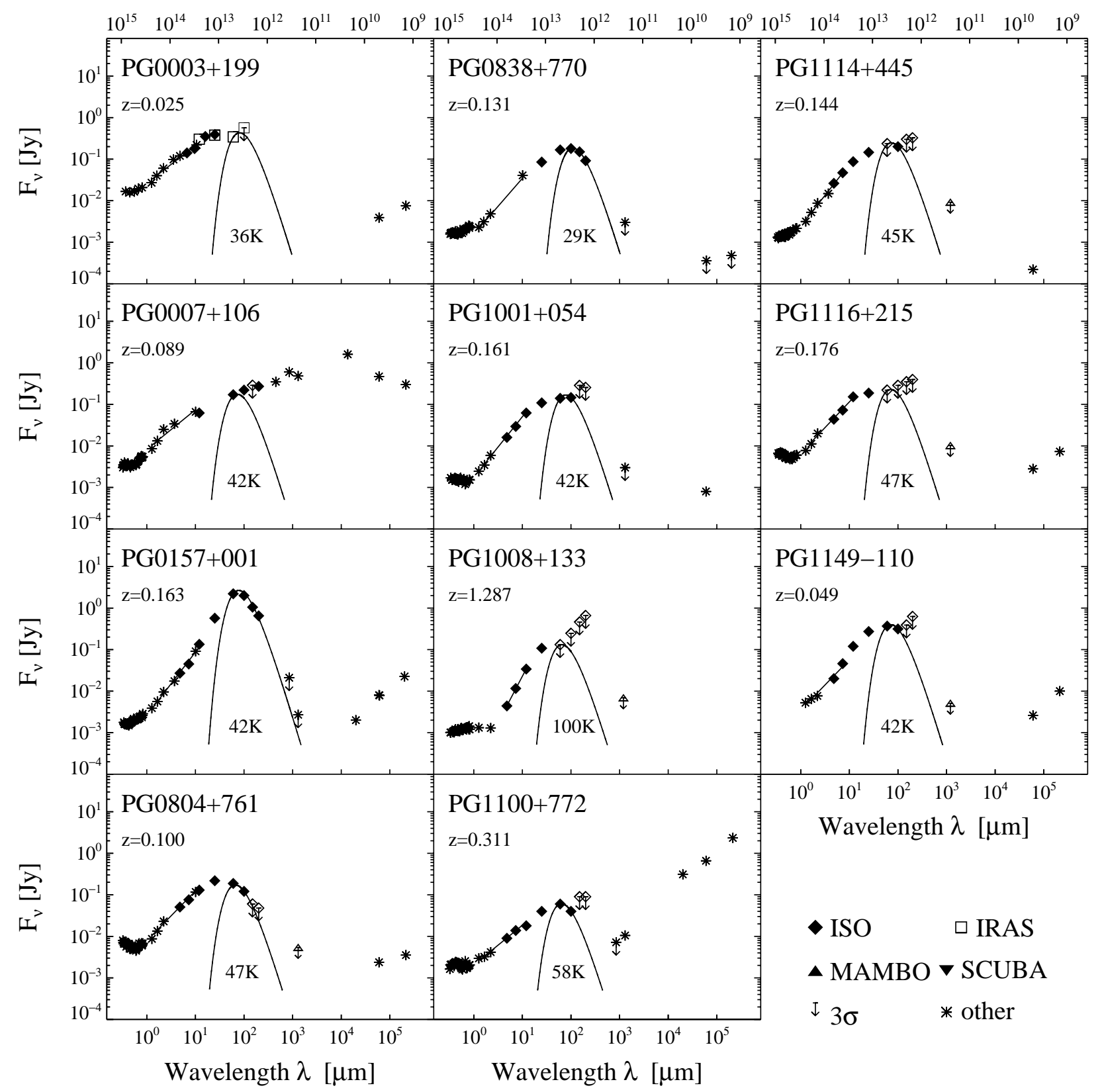

Fig. 1. Spectral energy distributions of 47 PG quasars (not including those of Paper I). The wavelength and frequency ranges are as observed and not corrected to the rest frame of the objects. Several modified blackbodies with dust emissivity proportional to $\lambda^{-2}$ are interactively fitted to the data and only the coolest component is plotted. The temperatures listed are corrected for redshift. Data from the literature (asterisks) are taken from Neugebauer et al. (1987) and de Vaucouleurs et al. (1991) at optical and NIR wavelengths, from Chini et al. (1989a, b) at mm wavelengths, and Kellermann et al. (1989) and the NVSS archive at radio wavelengths. Where ISO data coverage is sparse (e.g. for PG $0003+199$ beyond $25 \mu \mathrm{m}$ ) or the source is variable (PG1226+023), IRAS flux values from Sanders et al. (1989) are shown. The line between 1 and $10 \mu \mathrm{m}$ (in the quasar's restframe) shows the power law slope $\alpha_{\mathrm{IR}}$ as listed in Table 2.

symbols in Fig. 1 are small, compared to the range in wavelength and flux, which span typically more than two orders of magnitude. The most remarkable feature of the SEDs is the steady increase in flux density from near- to far-IR wavelengths with a peak at typically $25-60 \mu \mathrm{m}$, followed by a steep drop in most cases. The FIR-submm spectral index is well constrained for PG 0157+001, PG 1440+356 and PG 2130+099 with $\alpha_{200-850 \mu \mathrm{m}} \gtrsim 2.4,2.9$ and 2.6, respectively. (Note that $\alpha_{200-850 \mu \mathrm{m}}$ is also steep ( $\left.\gtrsim 2.6\right)$ for the 4 sources of Paper I where it is measured.) Since this seems to be too steep for standard models of synchrotron emission $\left(\alpha_{200-850 \mu \mathrm{m}} \lesssim 2\right)$, we suggest a thermal nature of the FIR emission for these and most of the other sources, which may show a less extreme spectral 


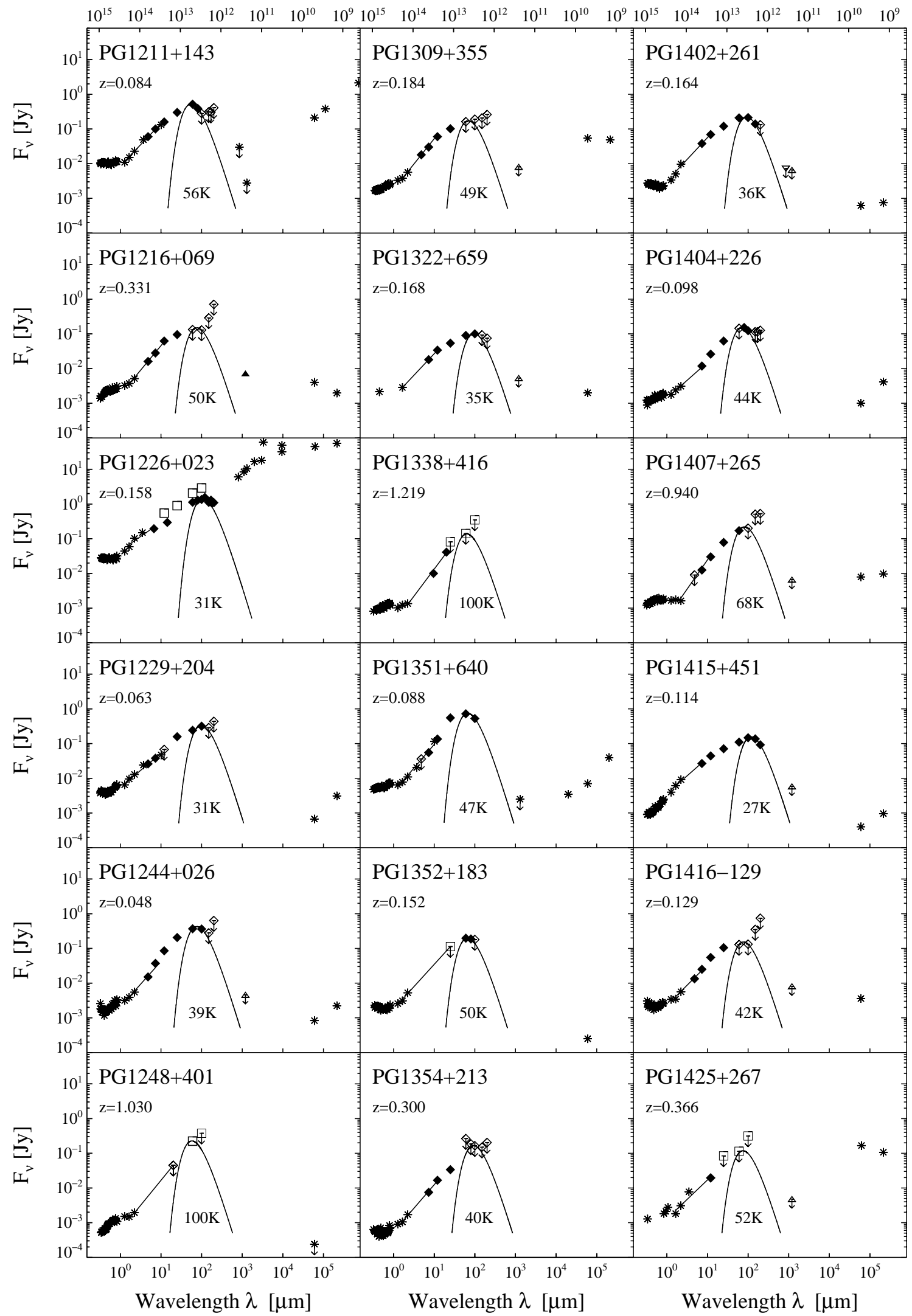

Fig. 1. continued. 


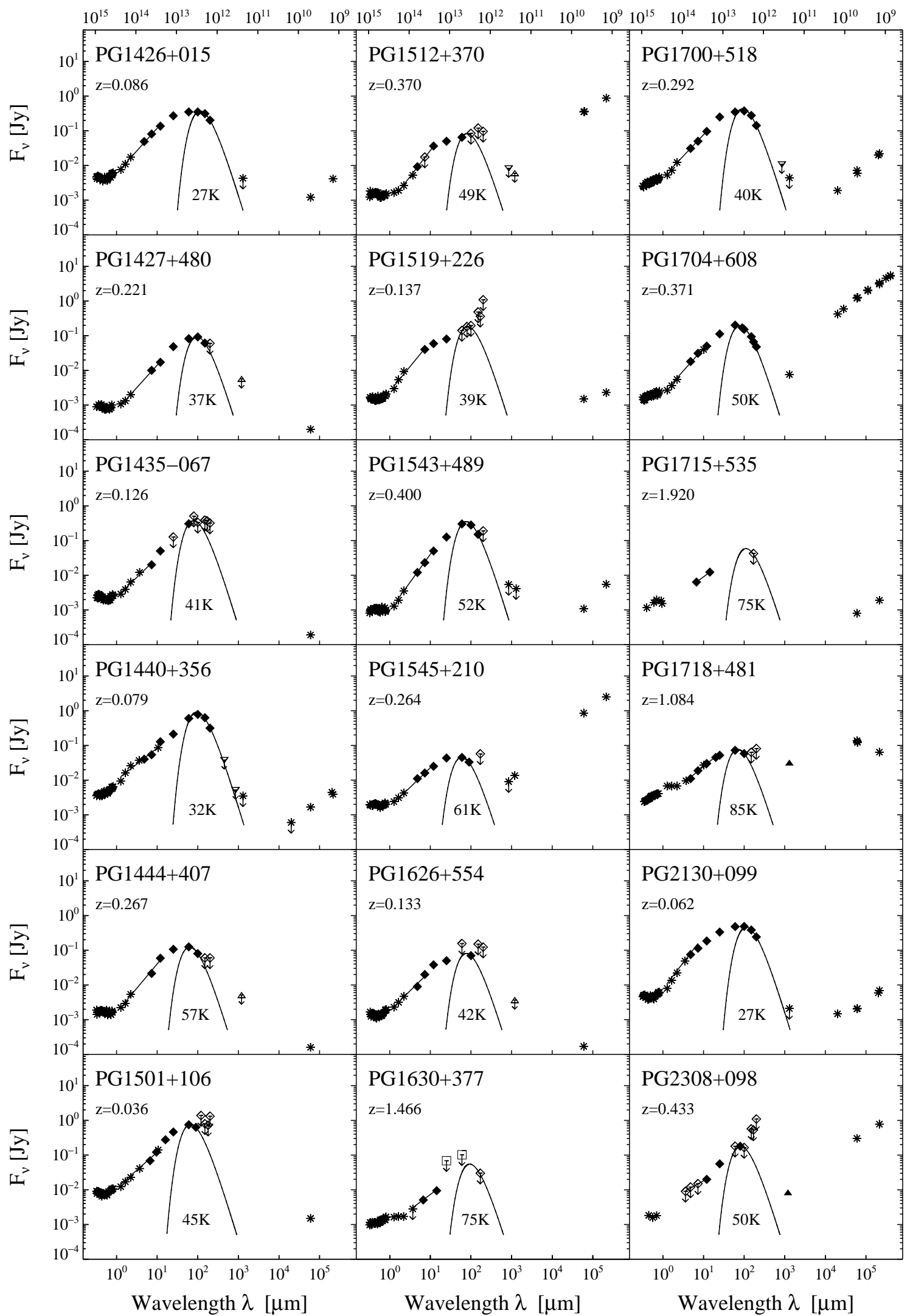

Fig. 1. continued. 

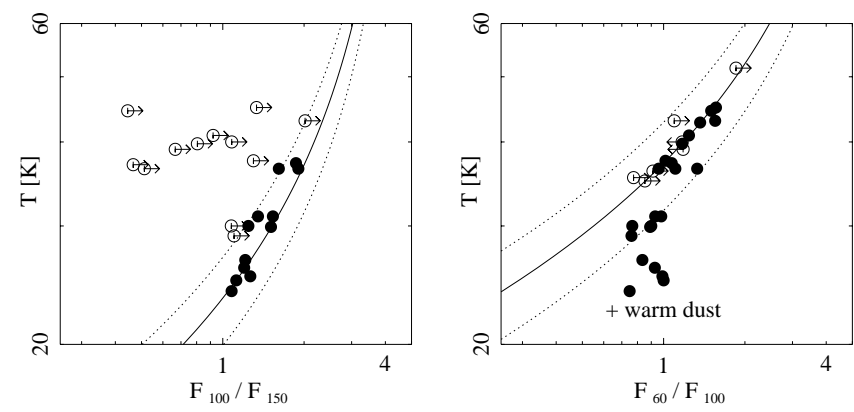

Fig. 2. FIR flux ratios and temperatures: The variation for a modified blackbody is shown by the solid line, the dotted lines indicate the range $T \pm 15 \%$. The symbols show the temperature of the coolest dust component fitted as shown in Fig. 1, but with $T$ values in the observers frame $(\bullet=$ based on detections, $\circ=$ based on upper limits). For the detections the $F_{100} / F_{150}$ points and most of the $F_{60} / F_{100}$ data lie close to the drawn line, demonstrating the essential equivalence of both methods. Some sources exhibit a higher $F_{60} / F_{100}$ ratio due to additional warm dust which provides the $F_{60}$ excess (e.g. PG $0838+770$ ).

drop, but an obvious drop in flux density between FIR and radio wavelengths.

In order to discuss the SEDs in the framework of thermal emission, we have modelled the $3-1000 \mu \mathrm{m}$ IR SED of each quasar by a superposition of several blackbodies with dust emissivity index $\beta=2$, indicating the presence of a wide variety of temperatures from hot $(1000 \mathrm{~K})$ to cool $(30 \mathrm{~K})$ dust in these objects. Since automatic multiple blackbody fits are neither unique nor stable, we have used an interactively supervised fit of several (3-8) components. For the purpose of lucidity we have plotted only the coolest component in Fig. 1. Its temperature (corrected for redshift) lies between 30 and $100 \mathrm{~K}$. This method of describing the cold FIR-submm end of the thermal spectrum is, in principle, equivalent to the use of FIR colours (e.g. $F_{100} / F_{150}$ ), as illustrated in Fig. 2 . While the simple flux ratio cannot account for the redshift effects, which are essential, our redshift corrected temperatures have the advantage to homogenize the sample. Despite the ambiguity involved in the multiple Planckian fits, we therefore prefered the lowest possible temperature fit over the simple $F_{100} / F_{150}$ method. The fits are well constrained, if the decline of the FIR or submm fluxes is clear (for example in PG 0157+001 and PG 0838+770); in this case the uncertainty of $T$ is about $15 \ldots 30 \%$, i.e. $5 \ldots 10 \mathrm{~K}$. The cold end of the thermal spectrum is less constrained, if the SED peaks in the MIR with a somewhat shallow decline to the FIR (for example in PG 0804+761). For the 16 cases of high FIR/submm upper limits, the coolest component is chosen so that both its temperature and the SED shape resembles that of the well measured sources; nevertheless the uncertainty of $T$ could reach up to $50 \%$, i.e. $T=50 \mathrm{~K}$ instead of an estimated value of $T \approx 100 \mathrm{~K}$. For example, PG 1001+054 or PG $1008+133$ could contain a colder component than that shown, but then an unusually constant FIR flux plateau would be required. The effect of the upper limits is discussed in Sect. 4.1.

As an alternative to the superposition of several hot and warm blackbodies, the rise of the SEDs between 1 and $10 \mu \mathrm{m}$ can be fitted by a power law $F_{v} \propto v^{\alpha}$ as shown by the

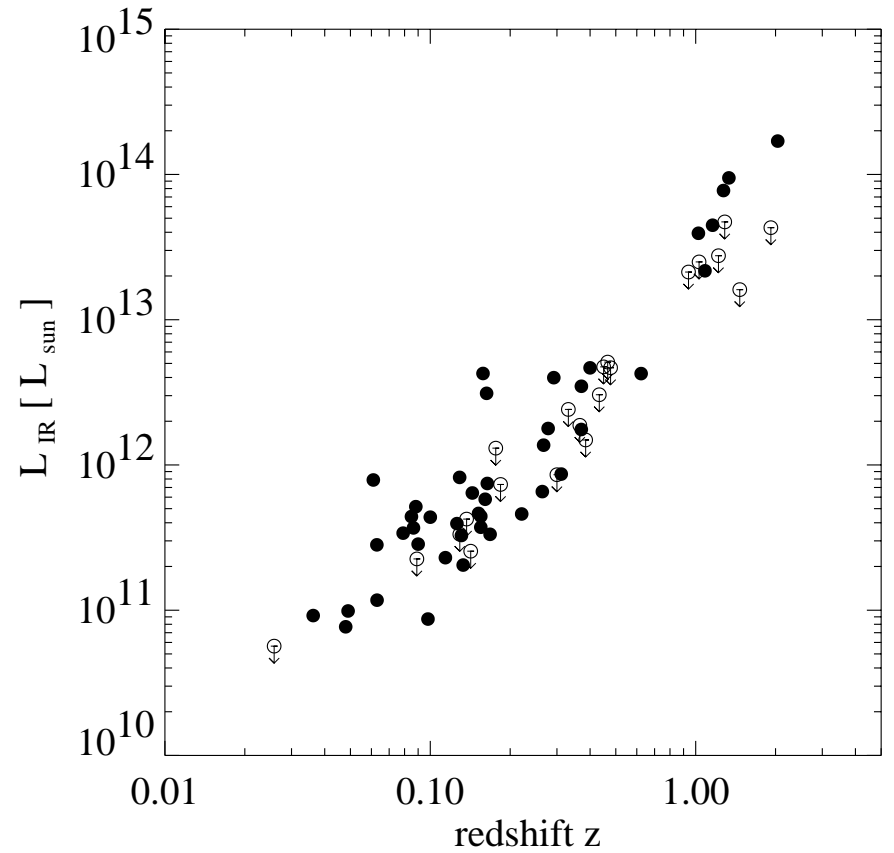

Fig. 3. Infrared luminosity (from 3 to $1000 \mu \mathrm{m}$ in the object restframe) against redshift. The symbols are: $\bullet=$ based on detections, $\circ=$ based on upper limits.

straight lines in Fig. 1. The actual SEDs, however, deviate from this idealised description by small bumps and curvatures. For the entire sample of 64 sources, the fitted spectral index $\alpha$ ranges from -0.9 ("flat") to -2.2 ("steep"), with a mean of $-1.3 \pm 0.3$. For comparison, the near- to mid-infrared SEDs of the four warm ULIRGs IRAS 05189-2524, Mrk 231, Mrk 463 and IRAS 19254-7245 (Klaas et al. 2001) can also be fitted by a power law with a mean $\alpha=-1.6 \pm 0.05$, which lies at the steep end of the slope distribution for the PG quasars.

For all 64 sources Table 2 lists the derived parameters like luminosities, dust temperatures and masses, and the values of $\alpha$ used in the discussion of the entire sample.

\section{Discussion}

The results of the full sample confirm the former findings for the 17 sources reported in Paper I. Therefore, we do not repeat here the discussion in Paper I, but rather concentrate on a few topics, on which the higher quality and better statistics of the larger sample has an impact.

\subsection{Luminous dust emission}

The spectra shown in Fig. 1 provide an impressive confirmation of the fact that the SED of an optically selected quasar contains a mid-far infrared bump due to thermal emission by dust. The thermal infrared luminosities of our sample span three orders of magnitude, from luminous $10^{11} L_{\odot}$, to ultraluminous $10^{12} L_{\odot}$, to hyperluminous $10^{13}-10^{14} L_{\odot}$ objects (Table 2). The most luminous objects lie at the highest redshift (Fig. 3), which is typical for flux limited samples (like the PG catalog). For those quasars for which we could establish only upper limits the possible far-infrared dust emission exceeds $10^{11} L_{\odot}$, except for the 
Table 2. Luminosities, spectral slopes, dust temperatures and masses, host morphologies and SED classes of the PG quasars discussed in this paper, including the sources of Paper I listed below the horizontal line. The soft X-ray $0.1-2.4 \mathrm{keV}$ luminosity $L_{\mathrm{X}}$ is derived from the ROSAT archive using the standard conversion of $10^{-11} \mathrm{erg} / \mathrm{cm}^{2}$ per ROSAT PSPC count, and $2.42 \times 10^{-6} \mathrm{erg} / \mathrm{cm}^{2} \mathrm{~s} \mathrm{keV}$ (priv. comm. by G. Hasinger); the resulting $L_{X}$ are within $50 \%$ of those listed by Laor et al. (1997) for 18 sources in common. In the restframe of the quasar the X-ray window shifts according to $z$. The absolute blue luminosity $L_{\mathrm{B}}$ in the restframe of the quasar is taken from Véron-Cetty \& Véron (1998). The infrared luminosities $L_{\mathrm{NIR}}(3-10 \mu \mathrm{m}), L_{\mathrm{MIR}}(10-40 \mu \mathrm{m}), L_{\mathrm{FIR}}(40-150 \mu \mathrm{m})$ and $L_{\text {submm }}(150-1000 \mu \mathrm{m})$ are computed for the restframe of the quasars. Note that $L_{\text {submm }}$ is mostly an extrapolation derived from the Rayleigh-Jeans tail of the cold blackbody shown in Fig. 1. $L_{1.4} \mathrm{GHz}$ is retrieved from the NVSS archive. The spectral index $\alpha_{\mathrm{X}}$ has been determined from ASCA by George et al. (2000). $\alpha_{\mathrm{opt}}$ and $\alpha_{\mathrm{IR}}$ refer to $F_{v}=v^{\alpha}$ fitted in the restframe of the quasar (with an uncertainty of about \pm 0.2 ); a "?" marks uncertain fits. $T_{\text {dust }}$ refers to the coldest dust component shown in Fig. 1 , the uncertainty is $5-10 \mathrm{~K}$ and can raise up to $50 \%$ in cases of poor FIR upper limits. The dust mass $M_{\text {dust }}$ is estimated according to Formula 6 in Stickel et al. (2000) following Hildebrand (1983), with uncertainties up to an order of magnitude. For the columns $T_{\text {dust }}$ and $M_{\text {dust }}$ a “ $\sim$ marks uncertain values due to upper limits of the flux densities. Two types of host morphology are adopted; disturbed (1) and undisturbed (0) as listed in Clements (2000) and derived from Surace et al. (2001). The SED classes assigned refer to Fig. 12.

\begin{tabular}{|c|c|c|c|c|c|c|c|c|c|c|c|c|c|c|c|c|}
\hline PG & $z$ & $\begin{array}{c}D_{\mathrm{L}} \\
\mathrm{Mpc} \\
\end{array}$ & $\begin{array}{r}L_{\mathrm{X}} \\
10^{9} L_{\odot} \\
\end{array}$ & $\begin{array}{r}L_{\mathrm{B}} \\
10^{9} L_{\odot} \\
\end{array}$ & $\begin{array}{r}L_{\mathrm{NIR}} \\
10^{9} L_{\odot} \\
\end{array}$ & $\begin{array}{r}L_{\mathrm{MIR}} \\
10^{9} L_{\odot} \\
\end{array}$ & $\begin{array}{r}L_{\mathrm{FIR}} \\
10^{9} L_{\odot} \\
\end{array}$ & $\begin{array}{l}L_{\text {submm }} \\
10^{9} L_{\odot} \\
\end{array}$ & $\begin{array}{c}L_{1.4 \mathrm{GHz}} \\
\log L_{\odot} \\
\end{array}$ & $\begin{array}{r}\alpha_{\mathrm{X}} \\
2-10 \mathrm{keV} \\
\end{array}$ & $\begin{array}{r}\alpha_{\mathrm{opt}} \\
0.3-1 \mu \mathrm{m} \\
\end{array}$ & $\begin{array}{r}\alpha_{\mathrm{IR}} \\
1-10 \mu \mathrm{m} \\
\end{array}$ & $\begin{array}{r}T_{\text {dust }} \\
\mathrm{K} \\
\end{array}$ & $\begin{array}{r}M_{\text {dust }} \\
10^{5} M_{\odot} \\
\end{array}$ & $\begin{array}{c}\text { host } \\
\text { morph }\end{array}$ & $\begin{array}{l}\text { SED } \\
\text { class } \\
\end{array}$ \\
\hline $0003+199$ & 0.025 & 104 & 20 & 29 & 27 & 22 & $<6$ & $<0.34$ & 10.58 & 1.94 & -0.65 & -0.90 & $\sim 36$ & $\sim 9$ & & $3 c$ \\
\hline $0007+106$ & 0.089 & 371 & 28 & 42 & 124 & 70 & $<29$ & $<1.05$ & 12.78 & & -0.23 & -0.95 & $\sim 42$ & $\sim 18$ & 1 & \\
\hline $0157+001$ & 0.163 & 704 & 62 & 343 & 392 & 1270 & 1395 & 53.78 & 12.78 & & -0.50 & -1.51 & 42 & 943 & 1 & 2 \\
\hline $0804+761$ & 0.100 & 419 & 120 & 146 & 230 & 180 & 39 & 0.95 & 11.44 & 2.18 & 0.43 & -1.20 & 47 & 13 & 0 & $3 c$ \\
\hline $0838+770$ & 0.131 & 557 & 55 & 79 & 94 & 133 & 73 & 6.11 & 10.83 & & -0.26 & -1.35 & 29 & 266 & 0 & $3 a$ \\
\hline $1001+054$ & 0.161 & 695 & $2^{*}$ & 111 & 233 & 251 & 92 & 3.31 & $11.63^{+}$ & & 0.03 & -1.46 & 42 & 59 & 0 & $3 a$ \\
\hline $1008+133$ & 1.287 & 8454 & & 10088 & 21115 & 23784 & $<2317$ & $<11.74$ & & & 0.09 & -2.24 & $\sim 100$ & $\sim 47$ & & $3 b$ \\
\hline $1100+772$ & 0.311 & 1439 & 260 & 724 & 388 & 360 & 114 & 1.71 & 15.33 & & 0.21 & -0.96 & 58 & 16 & 0 & $3 c$ \\
\hline $1114+445$ & 0.144 & 617 & 24 & 278 & 245 & 283 & 108 & 3.31 & $10.96^{+}$ & 1.51 & -0.56 & -1.45 & 45 & 53 & 1 & $3 a$ \\
\hline $1116+215$ & 0.176 & 767 & 460 & 529 & 585 & 563 & $<151$ & $<4.03$ & 12.27 & 2.06 & -0.05 & -1.27 & $\sim 47$ & $\sim 57$ & & $3 c$ \\
\hline $1149-110$ & 0.049 & 200 & 17 & 23 & 23 & 54 & 20 & 0.76 & 11.27 & & & -1.21 & 42 & 14 & & \\
\hline $1211+143$ & 0.084 & 353 & 130 & 160 & 186 & 179 & 73 & 1.24 & 11.44 & 2.00 & -0.11 & -1.22 & 56 & 13 & 1 & $3 c$ \\
\hline $1216+069$ & 0.331 & 1543 & 500 & 1009 & 991 & 1073 & $<339$ & $<7.73$ & 12.71 & 1.76 & -0.50 & -1.41 & $\sim 50$ & $\sim 99$ & & $3 a$ \\
\hline $1226+023$ & 0.158 & 681 & 2000 & 2753 & 2080 & 1355 & 767 & 60.72 & 16.06 & & -0.22 & $?-0.94$ & 31 & 2303 & 0 & $3 c$ \\
\hline $1229+204$ & 0.063 & 259 & 29 & 84 & 42 & 46 & 26 & 2.33 & 10.96 & & -0.50 & -0.98 & 31 & 88 & 0 & $3 c$ \\
\hline $1244+026$ & 0.048 & 196 & 34 & 12 & 15 & 39 & 20 & 0.92 & 10.57 & 2.46 & -0.58 & -1.31 & 39 & 19 & & $3 a$ \\
\hline $1248+401$ & 1.030 & 6237 & 1200 & 4404 & 7224 & $<15319$ & $<2440$ & $<12.38$ & & & -0.40 & $?-1.43^{a}$ & $\sim 100$ & $\sim 50$ & & \\
\hline $1309+355$ & 0.184 & 803 & 55 & 278 & 275 & 334 & $<121$ & $<2.95$ & 13.14 & & -0.56 & -1.40 & $\sim 49$ & $\sim 40$ & 0 & $3 a$ \\
\hline $1322+659$ & 0.168 & 727 & 220 & 175 & 126 & 139 & 63 & 3.65 & $11.06^{+}$ & 1.63 & & -1.24 & 35 & 100 & 0 & $3 a$ \\
\hline $1338+416$ & 1.219 & 7842 & & 8391 & 8779 & 15803 & $<2111$ & $<10.96$ & & & -0.08 & -1.56 & $\sim 100$ & $\sim 44$ & & $3 b$ \\
\hline $1351+640$ & 0.088 & 368 & & 20 & 119 & 273 & 119 & 3.36 & 12.32 & & -0.35 & -1.36 & 47 & 49 & 0 & 2 \\
\hline $1352+183$ & 0.152 & 653 & 88 & 211 & 162 & $<205$ & 93 & 2.17 & $11.07^{+}$ & & -0.22 & $?-1.28^{b}$ & $\sim 50$ & $\sim 28$ & 0 & $3 c$ \\
\hline $1354+213$ & 0.300 & 1379 & 140 & 440 & 215 & 332 & $<295$ & $<13.33$ & & & -0.32 & -1.36 & $\sim 40$ & $\sim 269$ & 0 & $3 a$ \\
\hline $1402+261$ & 0.164 & 709 & 200 & 253 & 314 & 289 & 134 & 6.97 & 11.22 & & 0.15 & -1.31 & 36 & 180 & 1 & $3 c$ \\
\hline $1404+226$ & 0.098 & 410 & 50 & 70 & 18 & 26 & 29 & 0.99 & 11.48 & 1.75 & -0.59 & -1.04 & 44 & 16 & 0 & 4 \\
\hline $1407+265$ & 0.940 & 5523 & 1900 & 6365 & 6595 & 11174 & $<3504$ & $<37.60$ & 14.11 & 2.05 & -0.11 & -1.68 & $\sim 68$ & $\sim 274$ & & $3 b$ \\
\hline $1415+451$ & 0.114 & 481 & 74 & 160 & 100 & 84 & 40 & 4.85 & 10.99 & & -0.90 & -1.03 & 27 & 274 & 0 & $3 a$ \\
\hline $1416-129$ & 0.129 & 549 & 59 & 64 & 115 & 161 & $<54$ & $<2.03$ & $12.07^{+}$ & 1.79 & 0.69 & -1.17 & $\sim 42$ & $\sim 37$ & 0 & $3 c$ \\
\hline $1425+267$ & 0.366 & 1730 & & 1202 & 613 & 932 & $<322$ & $<6.67$ & 14.15 & & & -1.12 & $\sim 52$ & $\sim 79$ & & \\
\hline $1426+015$ & 0.086 & 360 & 86 & 106 & 136 & 158 & 66 & 6.41 & 11.37 & & 0.14 & -1.22 & 27 & 351 & 1 & $3 a$ \\
\hline $1427+480$ & 0.221 & 981 & 110 & 77 & 122 & 199 & 100 & 5.20 & $10.32^{+}$ & & -0.13 & -1.27 & 37 & 129 & & $3 a$ \\
\hline $1435-067$ & 0.126 & 535 & 31 & 92 & 102 & 168 & 119 & 4.96 & $10.77^{+}$ & & 0.32 & -1.12 & $\sim 41$ & $\sim 96$ & 0 & $3 a$ \\
\hline $1440+356$ & 0.079 & 328 & 410 & 88 & 105 & 119 & 105 & 8.69 & 11.39 & 2.06 & -0.64 & -0.92 & 32 & 292 & 1 & 4 \\
\hline $1444+407$ & 0.267 & 1211 & 280 & 765 & 517 & 678 & 170 & 2.76 & $11.41^{+}$ & 2.21 & -0.24 & -1.40 & 57 & 27 & & $3 a$ \\
\hline $1501+106$ & 0.036 & 147 & 12 & 18 & 25 & 44 & 21 & 0.66 & $10.55^{+}$ & 1.70 & 0.10 & -1.03 & 45 & 10 & & $3 c$ \\
\hline $1512+370$ & 0.370 & 1756 & 410 & 937 & 817 & 698 & 233 & 5.87 & 15.13 & & -0.01 & -1.51 & $\sim 49$ & $\sim 79$ & & $3 a$ \\
\hline $1519+226$ & 0.137 & 585 & 44 & 58 & 209 & 151 & $<70$ & $<3.18$ & 11.54 & & -0.46 & -1.08 & $\sim 39$ & $\sim 68$ & 0 & $3 a$ \\
\hline $1543+489$ & 0.400 & 1918 & 200 & 529 & 1408 & 2180 & 1136 & 23.56 & 12.95 & 2.80 & -0.07 & -1.62 & 52 & 276 & & $3 a$ \\
\hline $1545+210$ & 0.264 & 1196 & 250 & 432 & 314 & 281 & 57 & 0.81 & 15.00 & & 0.07 & -1.12 & 61 & 7 & & $3 c$ \\
\hline $1626+554$ & 0.133 & 566 & 120 & 121 & 87 & 86 & 29 & 1.11 & $10.78^{+}$ & & -0.30 & -1.15 & 42 & 20 & 0 & $3 c$ \\
\hline $1630+377$ & 1.466 & 10155 & & 11062 & 7899 & 6163 & $<2072$ & $<17.79$ & & & -0.16 & -0.88 & $\sim 75$ & $\sim 110$ & & $3 \mathrm{c}$ \\
\hline $1700+518$ & 0.292 & 1337 & & 839 & 1322 & 1857 & 778 & 32.54 & 13.24 & & -0.65 & -1.24 & 40 & 657 & 1 & 4 \\
\hline $1704+608$ & 0.371 & 1758 & & 1000 & 1373 & 1525 & 569 & 12.49 & 15.62 & & -0.46 & -1.31 & 50 & 159 & & $3 a$ \\
\hline $1715+535$ & 1.920 & 15042 & & 23111 & 19694 & 19185 & $<4109$ & $<35.32$ & 14.27 & & & -0.87 & $\sim 75$ & $\sim 218$ & & \\
\hline $1718+481$ & 1.084 & 6681 & 1800 & 33406 & 12162 & 8296 & 1248 & 8.22 & 15.10 & & -0.45 & -0.92 & 85 & 42 & & $3 c$ \\
\hline $2130+099$ & 0.062 & 259 & 53 & 78 & 125 & 106 & 45 & 4.31 & 11.31 & & 0.09 & -1.52 & 27 & 239 & 1 & $3 a$ \\
\hline $2308+098$ & 0.433 & 2107 & 460 & 1127 & 1013 & 1278 & $<736$ & $<16.01$ & 15.18 & & $?-0.71$ & $?-0.63$ & $\sim 50$ & $\sim 201$ & & \\
\hline
\end{tabular}


Table 2. continued.

\begin{tabular}{|c|c|c|c|c|c|c|c|c|c|c|c|c|c|c|c|c|}
\hline PG & $z$ & $\begin{array}{c}D_{\mathrm{L}} \\
\mathrm{Mpc}\end{array}$ & $\begin{array}{r}L_{\mathrm{X}} \\
10^{9} L_{\odot} \\
\end{array}$ & $\begin{array}{r}L_{\mathrm{B}} \\
10^{9} L_{\odot} \\
\end{array}$ & $\begin{array}{r}L_{\mathrm{NIR}} \\
10^{9} L_{\odot} \\
\end{array}$ & $\begin{array}{r}L_{\mathrm{MIR}} \\
10^{9} L_{\odot} \\
\end{array}$ & $\begin{array}{r}L_{\mathrm{FIR}} \\
10^{9} L_{\odot} \\
\end{array}$ & $\begin{array}{l}L_{\text {submm }} \\
10^{9} L_{\odot} \\
\end{array}$ & $\begin{array}{l}L_{1.4 \mathrm{GHz}} \\
\log L_{\odot} \\
\end{array}$ & $\begin{array}{r}\alpha_{\mathrm{X}} \\
2-10 \mathrm{keV} \\
\end{array}$ & $\begin{array}{r}\alpha_{\mathrm{opt}} \\
0.3-1 \mu \mathrm{m} \\
\end{array}$ & $\begin{array}{r}\alpha_{\mathrm{IR}} \\
1-10 \mu \mathrm{m} \\
\end{array}$ & $\begin{array}{r}T_{\text {dust }} \\
\mathrm{K} \\
\end{array}$ & $\begin{array}{r}M_{\text {dust }} \\
10^{5} M_{\odot} \\
\end{array}$ & $\begin{array}{c}\text { host } \\
\text { morph }\end{array}$ & $\begin{array}{l}\text { SED } \\
\text { class }\end{array}$ \\
\hline $0003+158$ & 0.450 & 2203 & 812 & 765 & $<2149$ & $<2072$ & $<499$ & $<15.80$ & 15.24 & & -0.08 & $?-1.60$ & $\sim 44$ & $\sim 268$ & & \\
\hline $0026+129$ & 0.142 & 608 & 108 & 160 & $<124$ & $<74$ & $<53$ & $<3.85$ & 12.08 & & -0.19 & $?-0.74$ & 34 & 111 & 0 & \\
\hline $0043+039$ & 0.385 & 1835 & $<364$ & 1009 & $<538$ & $<596$ & $<339$ & $<13.13$ & & & -0.12 & $?-1.16$ & $\sim 42$ & $\sim 245$ & & \\
\hline $0044+030$ & 0.623 & 3266 & 282 & 1753 & 1462 & 1681 & 1067 & 46.27 & 14.91 & & -0.12 & -1.25 & 41 & 897 & & $3 \mathrm{c}$ \\
\hline $0050+124$ & 0.061 & 251 & 35 & 92 & 221 & 339 & 207 & 21.25 & 11.42 & 2.25 & -0.41 & -1.21 & 29 & 983 & 1 & $3 a$ \\
\hline $0052+251$ & 0.155 & 668 & 149 & 253 & 215 & 154 & 66 & 8.11 & $11.56^{+}$ & & -0.19 & -1.05 & $\sim 27$ & $\sim 465$ & 1 & $3 \mathrm{c}$ \\
\hline $1206+459$ & 1.158 & 7309 & $<3703$ & 14582 & 11200 & 24526 & 8891 & 96.44 & $12.85^{+}$ & & -0.41 & -2.02 & 67 & 723 & & $3 b$ \\
\hline $1241+176$ & 1.273 & 8327 & $<4563$ & 11062 & 25543 & 46870 & 5084 & 22.50 & 16.08 & & -0.71 & $?-1.83$ & 111 & 77 & & $3 b$ \\
\hline $1247+267$ & 2.038 & 16448 & 5978 & 58052 & 64160 & 98915 & 6349 & 23.31 & $14.47^{+}$ & 2.07 & -0.24 & -2.01 & 131 & 65 & & $3 b$ \\
\hline $1254+047$ & 1.024 & 6189 & $<2831$ & 6979 & 12454 & 21024 & 4857 & 32.56 & $13.05^{+}$ & & -0.11 & -1.93 & 85 & 167 & & $3 b$ \\
\hline $1259+593$ & 0.478 & 2367 & $<567$ & 1599 & $<2210$ & $<1962$ & $<494$ & $<4.32$ & $11.26^{+}$ & & -0.08 & $?-1.46$ & $\sim 74$ & $\sim 28$ & & $3 a$ \\
\hline 1302-102 & 0.278 & 1266 & 318 & 1106 & 431 & 714 & 621 & 16.78 & 14.71 & & -0.07 & -0.94 & 47 & 239 & 1 & $3 \mathrm{c}$ \\
\hline $1307+085$ & 0.155 & 668 & 169 & 278 & 124 & 141 & 105 & 2.52 & $11.23^{+}$ & & -0.19 & -0.81 & 50 & 32 & 0 & $3 \mathrm{c}$ \\
\hline $1411+442$ & 0.090 & 376 & $3^{*}$ & 121 & 144 & 111 & 29 & 1.07 & 11.18 & -0.42 & -0.54 & -1.19 & 41 & 20 & 1 & $3 b$ \\
\hline $1613+658$ & 0.129 & 549 & 164 & 101 & 181 & 304 & 299 & 37.27 & 11.81 & & -1.02 & -1.13 & 27 & 2013 & 1 & 4 \\
\hline $1634+706$ & 1.334 & 8889 & 1411 & 52944 & 36093 & 42572 & 12913 & 137.06 & 14.02 & 2.03 & -0.17 & -1.35 & 68 & 1002 & 0 & $3 b$ \\
\hline $2112+059$ & 0.466 & 2297 & 15 & 1213 & $<2425$ & $<1448$ & $<1141$ & $<93.98$ & 12.91 & & +0.02 & -1.06 & $\sim 32$ & $\sim 3177$ & & $3 c$ \\
\hline
\end{tabular}

* Value low due to steep $\alpha_{0.3-2 \mathrm{keV}}$ (Laor et al. 1997); ${ }^{+}$extrapolated from $5 \mathrm{GHz}$ using $F(1.4 \mathrm{GHZ}) \approx 2.5 \times F(5 \mathrm{GHZ}) ;{ }^{a}$ between 1 and $15 \mu \mathrm{m}$; ${ }^{b}$ between 1 and $25 \mu \mathrm{m}$.

low luminosity quasar PG 0003+199 (Mrk 335). As indicated in Fig. 1, a luminous dust component could also be placed underneath the SED of the flat spectrum radio-loud source PG 0007+106 which does not show any thermal bump. Since the PG quasars were selected optically, the high IR detection rate suggests that every quasar, irrespective of being optically visible or dust obscured, has a high inherent dust luminosity.

We have decomposed the IR emission into the NIR (3-10 $\mu \mathrm{m})$, MIR $(10-40 \mu \mathrm{m})$, FIR $(40-150 \mu \mathrm{m})$ and submm $(150-1000 \mu \mathrm{m})$ regimes (Table 2). Figure 4 shows that for each of these regimes the IR luminosity is correlated with the blue luminosity (Table 2). While the correlation is of similar quality for the NIR and MIR ranges and also for the FIR (Table 3), the larger scatter for the submm regime (Table 3 ) might also be due to the lack of enough data points to constrain $L_{\text {submm }}$ well, which therefore in most cases is just the blackbody extrapolation from the FIR. Also of interest is, that the soft $\mathrm{X}$-ray luminosity correlates to some extent with the NIR, MIR and FIR luminosity (Fig. 4, Table 3), whereby the scatter is partly caused by some extinguished sources with steep $\alpha_{0.3-2 \mathrm{keV}}$ (e.g. PG $1001+054$, PG 1411+442, PG 2112+059, Laor et al. 1997; Wang et al. 2000; Gallagher et al. 2001), and in the restframe of the quasar the X-ray window shifts according to $z$ (for the high redshift sources like PG 1247+267, PG 1634+706, PG 1718+481). An analysis of 2-10 keV ASCA spectra (George et al. 2000) indicates that the relative decline of $\mathrm{X}$-ray (to optical) luminosity with $z$ is probably due to both intrinsic properties of the sources (as favoured by Bechtold et al. 1994; Wilkes et al. 1994) and observational effects (proposed by Yuan et al. 1998). Because of the observational X-ray window limitation and in view of several other effects, we do not investigate the correlations in more detail. For the scope of our discussion we conclude, that the IR luminosities are correlated over four orders of magnitude with both the blue and the soft
Table 3. Correlation coefficients (Pearson's r) for luminosities shown in Fig. 4 and listed in Table 2. The correlations are derived for the logarithms of the luminosities.

\begin{tabular}{lrcccc}
\hline \hline & $L_{\mathrm{X}}$ & $L_{\mathrm{B}}$ & $L_{\mathrm{NIR}}$ & $L_{\mathrm{MIR}}$ & $L_{\mathrm{FIR}}$ \\
\hline$L_{\mathrm{B}}$ & 0.83 & & & & \\
$L_{\mathrm{NIR}}$ & 0.80 & 0.97 & & & \\
$L_{\mathrm{MIR}}$ & 0.79 & 0.94 & 0.98 & & \\
$L_{\mathrm{FIR}}$ & 0.79 & 0.89 & 0.93 & 0.96 & \\
$L_{\text {submm }}$ & 0.60 & 0.66 & 0.71 & 0.74 & 0.87 \\
\hline
\end{tabular}

$\mathrm{X}$-ray luminosity, which provide the primary measure of the AGN strength. But these correlations show also a considerable scatter with a factor of about three and five for $L_{\text {blue }}$ and $L_{\mathrm{X}}$, respectively.

The dust masses derived from the blackbodies shown in Fig. 1 lie between $10^{6}$ and $2 \times 10^{8} M_{\odot}$ (Table 2), i.e. are comparable to those in Seyfert 1 galaxies (from the $12 \mu \mathrm{m}$ AGN sample of Spinoglio et al. 2002) and ULIRGs (from Klaas et al. 2001). Figure 5 illustrates the distribution of FIR luminosities and dust masses. We used a dust emissivity $\beta=2$ throughout. If in the hyperluminous quasars due to the higher dust temperature $\beta$ changed, e.g. $\beta=1$, then their dust mass would be lower by a factor of about 2 . But this has no significant impact on the observed dust mass range. Rather the dust mass depends essentially on the temperature of the coldest component. This is uncertain in the case of upper limits for the FIR fluxes, so that the dust mass could be lower or - if the dust temperature is much colder - also higher (by up to an order of magnitude) than the estimates listed in Table 2.

For those sources with flux density upper limits at $\lambda>60 \mu \mathrm{m}$, it is not only of interest, how well the FIR luminosity and the temperature of the cold dust component are constrained, but also how well it is actually approximated. 

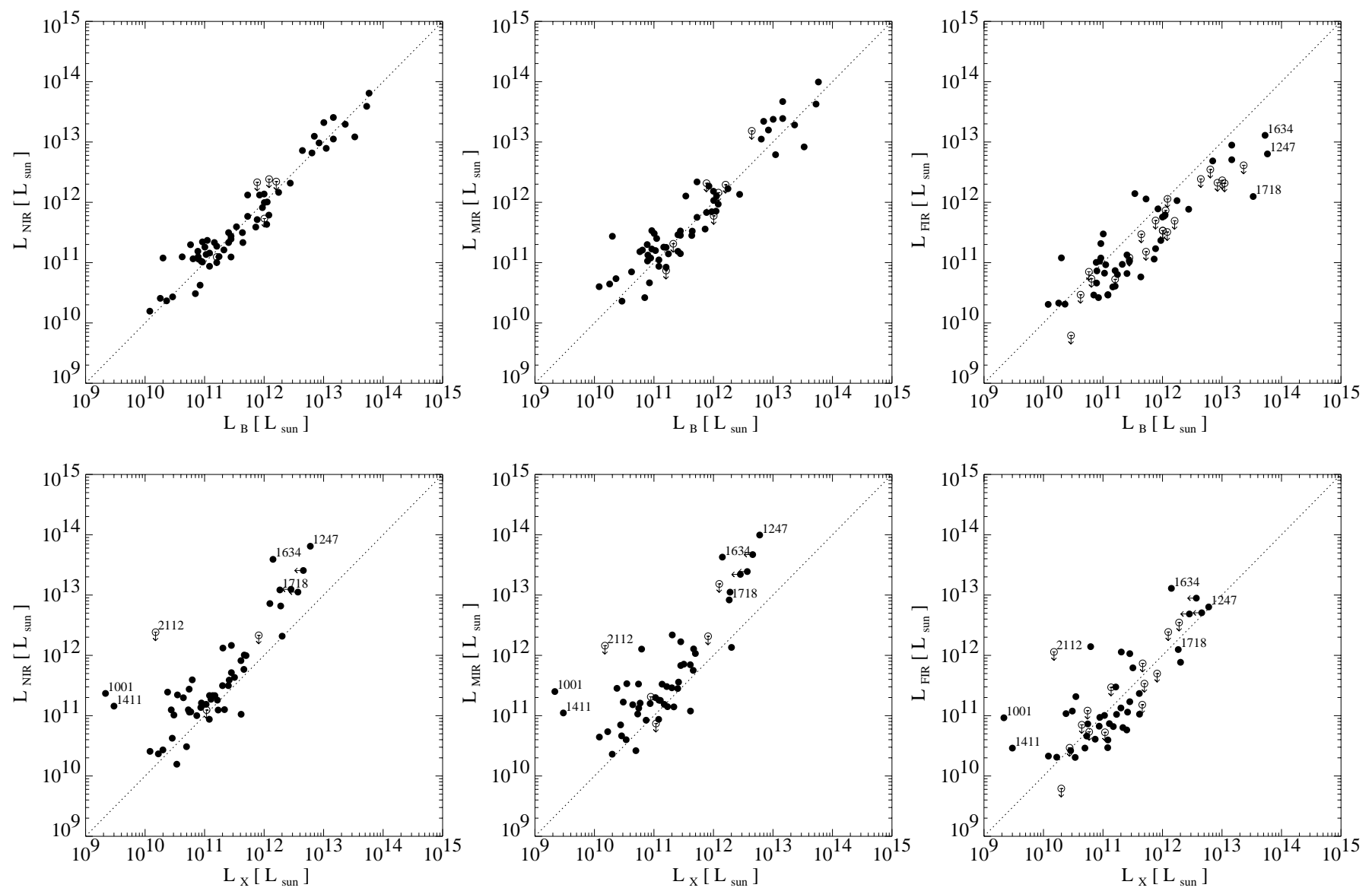

Fig. 4. Infrared versus blue and soft X-ray luminosities. The infrared is separated into the NIR (3-10 $\mu \mathrm{m})$, MIR (10-40 $\mu \mathrm{m})$ and FIR $(40-150 \mu \mathrm{m})$ ranges. The symbols are: $\bullet=$ based on detections, $\circ=$ based on upper limits. The dotted diagonal lines indicate unity.

Therefore we consider firstly the cases with good detections in at least 8 ISO filters (e.g. PG 0157+001, PG 1426+015, PG 1700+518, PG 1704+608, PG 2130+099): a visual inspection of the SED shapes shows that they run smoothly without any abrupt bents, suggesting that this is a general feature of a quasar SED. Now we consider both the height of the upper limits around the peak wavelength and how far the cold component could become even colder. If in the case of upper limits the sources at low/intermediate redshift $(z<0.4)$ possess SED shapes similar to the sources with FIR detections, then a visual inspection indicates that the measured $60-100 \mu \mathrm{m}$ upper limits are not far above the expectations (pictured by the cold blackbody in Fig. 1). For example, comparing the pairs PG 0804+761 - PG 1116+215 (at $z \approx 0.1$ ) or PG 1444+407 PG $1512+370$ (at $z \approx 0.3$ ), suggests that the peak of the flux density lies at a wavelength of about $60-80 \mu \mathrm{m}$ and reaches a value close to the upper limits measured. Otherwise, the SEDs of PG $1116+215$ or PG $1512+370$ would exhibit an abrupt decline longwards of $25 \mu \mathrm{m}$ or a broad FIR flux plateau, which would also be unusual, because it is not seen in (other brighter) sources with detections. Hence, we suggest that the FIR luminosity is in fact approximated within a factor of about 2 or better, i.e. the " <" sign in column $L_{\text {FIR }}$ of Table 2 can actually be replaced by a " $\approx$ " sign.

At high redshift $(0.9<z<2.0)$ the complete ISO PG sample contains 12 sources, out of which 6 (PG 1718+481 and 5 sources in Paper I) show a clear SED maximum at about $60-80 \mu \mathrm{m}$ in the observer's frame. Among the remaining 6 sources 2 SEDs are poorly constrained and omitted from the discussion (PG 1248+401 and PG 1338+416). For 2 sources (PG 1008+133 and PG 1407+265) the SED flattens, suggesting actually a maximum around $60 \mu \mathrm{m}$ (in the observers frame). And for 2 sources (PG 1630+377 and PG 1715+535) the $170 \mu \mathrm{m}$ upper limit restricts the range of the cold dust component: If it is warmer than the $75 \mathrm{~K}$ component shown in Fig. 1, e.g. $100 \mathrm{~K}$, then the MIR/FIR luminosity ratio becomes unusually high (as discussed below in Sect. 4.2.2); if it is colder, e.g. $50 \mathrm{~K}$, resulting in a peak around $170 \mu \mathrm{m}$, then the MIR luminosity declines, but (due to the $170 \mu \mathrm{m}$ upper limit) the FIR luminosity remarkably remains about the same as for a $75 \mathrm{~K}$ component. Again, as for the low/intermediate redshifted sources, we conclude that the FIR luminosity might be approximated by a factor of about 2 or better. The uncertainty in the temperature of the cold dust component of the $z>0.9$ sources is certainly higher.

Molecular gas masses (or upper limits) have been published by Barvainis et al. (1989), Solomon et al. (1997), Barvainis et al. (1998), Evans et al. (2001) from CO observations for some of our quasars. They are listed in Table 4. Comparing, for the good $\mathrm{CO}$ detections, the gas and dust masses yields a mean (median) gas-to-dust mass ratio of about $250 \pm 120$ (270). Since for the gas mass determination these authors used a CO-to- $\mathrm{H}_{2}$ 


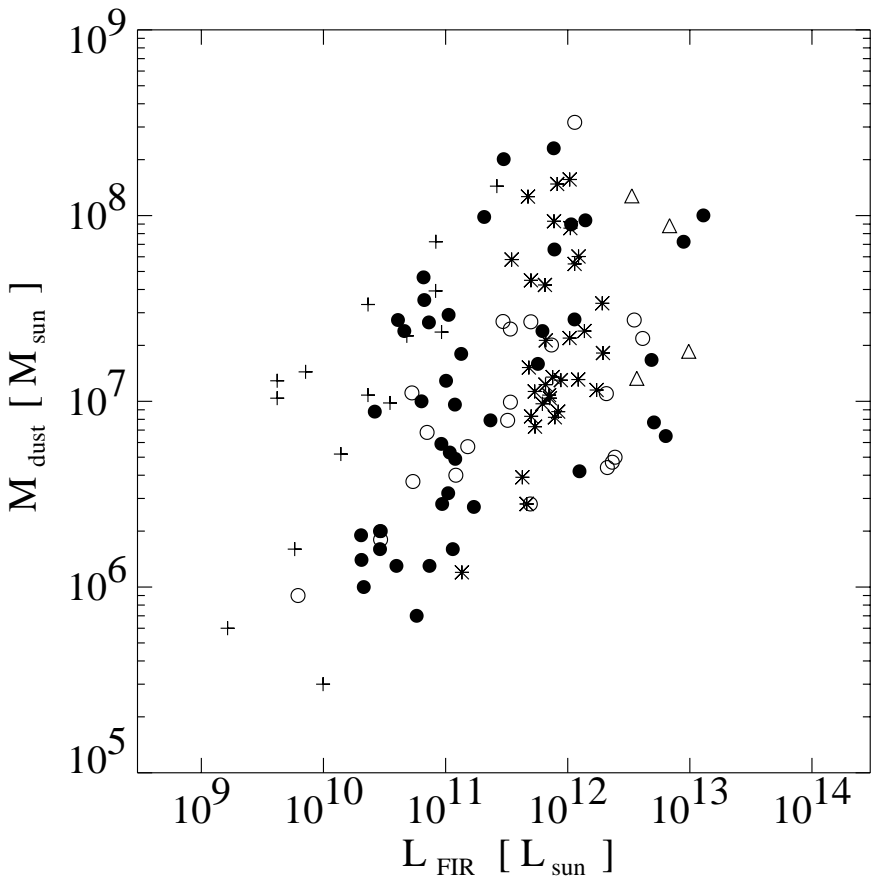

Fig. 5. Dust mass against FIR luminosity. The symbols are: $\bullet=$ based on detections, $\circ=$ based on upper limits. Note, that in case of FIR flux upper limits the dust mass does not necessarily drop, because the temperature could be lower than assumed; therefore no arrows are plotted. Also other samples are shown for comparison (with dust mass recalculated exactly as for the PG quasars): $*=$ ULIRGs from Klaas et al. (2001),$+=$ Seyfert 1 s from Spinoglio et al. (2002), $\Delta=$ HyLIGs from Verma et al. (2002, based on upper limits).

conversion factor of about $4 M_{\odot}\left(\mathrm{K} \mathrm{km} \mathrm{s}^{-1} \mathrm{pc}^{2}\right)^{-1}$, i.e. about a factor of 2 higher than the average galactic value, the mean (median) gas-to-dust mass ratio of the quasars can be considered as consistent with the canonical value of about 150 for normal spiral galaxies (e.g. Stickel et al. 2000). Note that this argues also in favour of the thermal nature of the IR emission and against a synchrotron origin. If we adopt this canonical value in general, then good upper limits of the gas mass could, in principle, also constrain the dust mass (and the temperature of the cold component). However, the available CO limits are not yet stringent enough. Concerning the mass and luminosity estimates for the cases of upper FIR flux limits, we conclude that the true FIR luminosities are approximated by a factor of about 2, while the dust mass estimates in Table 2 may be less constrained up to an order of magnitude.

\subsection{On the AGN-starburst connection}

The power-law rise of the IR SEDs indicates a well ordered dependence between the dust components at different temperatures. It suggests that the dust is heated by the central AGN (and not by sources which have an extended irregular spatial distribution), resulting in a smooth temperature gradient from inside to outside. While there is broad consensus that the strong MIR emission of PG quasars is due to the AGN, the debate is still on whether the FIR emission is mainly powered by the AGN, or whether starbursts are required as an additional energy source.
Table 4. Gas and dust masses (left panel), and PAH luminosity upper limits (right panel). The gas masses are from Barvainis et al. (1989), Solomon et al. (1997), Barvainis et al. (1998), Evans et al. (2001). The upper limits of the PAH $7.7 \mu \mathrm{m}$ feature are determined from the ISOPHOT-S spectra (Fig. 6).

\begin{tabular}{|c|c|c|c|c|c|c|}
\hline PG & $\begin{array}{r}M_{\text {dust }} \\
10^{5} M_{\odot}\end{array}$ & $\begin{array}{l}M\left(\mathrm{H}_{2}\right) \\
10^{7} M_{\odot}\end{array}$ & $\begin{array}{r}M\left(\mathrm{H}_{2}\right) / \\
M_{\mathrm{dust}}\end{array}$ & PG & $\begin{array}{r}F_{7.7 \mu \mathrm{m}} \\
{[\mathrm{mJy}]}\end{array}$ & $\begin{array}{r}L_{\mathrm{PAH}} / \\
L_{\mathrm{FIR}}\end{array}$ \\
\hline $0007+106$ & 18 & $<350$ & $<1940$ & $0003+199$ & $<25$ & $<0.55$ \\
\hline $0050+124$ & 983 & 1400 & 142 & $0050+124$ & $<30$ & $<0.88$ \\
\hline $0157+001$ & 943 & 4000 & 424 & $0157+001$ & $<50$ & $<0.08$ \\
\hline $0838+770$ & 266 & 840 & 316 & $0804+761$ & $<12$ & $<0.24$ \\
\hline $1206+459$ & 723 & $<4500$ & $<622$ & $1226+023$ & $<24$ & $<0.18$ \\
\hline $1241+176$ & 77 & $<2800$ & $<3640$ & $1501+106$ & $<30$ & $<0.38$ \\
\hline $1247+267$ & 65 & $<4500$ & $<6920$ & $1613+658$ & $<10$ & $<0.13$ \\
\hline $1254+047$ & 167 & $<4500$ & $<2690$ & $1700+518$ & $<20$ & $<0.55$ \\
\hline $1351+640$ & $>49$ & 520 & $<1060$ & $1704+608$ & $<25$ & $<1.66$ \\
\hline $1402+261$ & 180 & $<490$ & $<272$ & $2130+099$ & $<18$ & $<0.33$ \\
\hline $1415+451$ & 274 & 610 & 223 & & & \\
\hline $1440+356$ & 292 & 800 & 274 & & & \\
\hline $1613+658$ & 2013 & 2000 & 99 & & & \\
\hline $1634+706$ & 1002 & $<2200$ & $<220$ & & & \\
\hline
\end{tabular}

Decisive arguments implying starbursts as the main FIR power source are still searched for. We shall first consider, how far our data might strengthen the starburst case, and then see how they compare with the most actual AGN dust models.

\subsubsection{On the starburst tracers}

\subsubsection{Cool dust and starburst knots}

Since the large amount of dust must have been produced by stars, quasars are accompanied or preceeded by starbursts. Coeval starburst activity in quasars appears in some cases plausible from the detection of large amounts of molecular gas via $\mathrm{CO}$ observations (Table 4), since the vast dense gas reservoirs might be expected to be definitely converted into stars. For the luminous and ultraluminous quasars (but not for the hyperluminous ones discussed in Sect. 4.2.2) the temperature of the coolest dust component lies at about $30-50 \mathrm{~K}$, which is also the range found for nearby ultraluminous starburst galaxies (Klaas et al. 2001). Furthermore, some hosts of the - low luminosity - quasars show knotty morphological distortions (e.g. Surace et al. 2001) which are attributed to starbursts acting in the cooler or outer regions of these quasars.

\subsubsection{Constraints from $\mathrm{PAHs}$ ?}

There is general agreement that PAHs (Polycyclic Aromatic Hydrocarbonates, review by Léger \& Puget 1989), are distributed thoughout the galaxies and mixed with their ISM and dust (e.g. Haas et al. 2002 and references therein), and that they are destroyed (or transformed) in the hard radiation field of an AGN.

If the FIR (and submm) emission of quasars is mainly powered by starbursts, then a large fraction of the dusty volume is not effectively hit by the hard AGN radiation field. Then the PAH carriers mixed with the FIR emitting dust clouds should not be destroyed, hence this FIR emission should be 


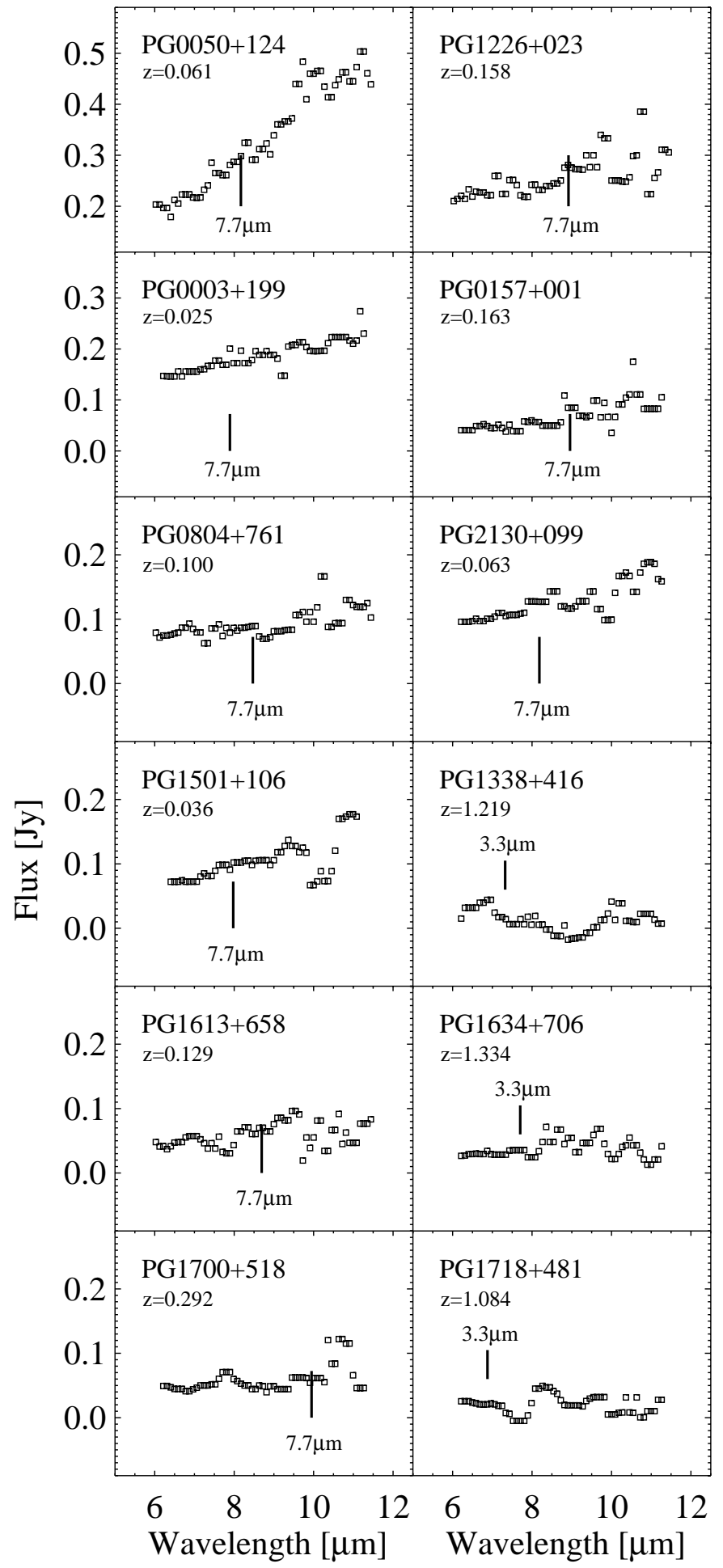

Fig. 6. MIR spectra of the 12 PG quasars observed with ISOPHOT-S. The $x$-axes give the wavelength as observed, the vertical bar denotes the $z$-dependent predicted position of the PAH features at 7.7 and $3.3 \mu \mathrm{m}$, respectively. At the low flux level the unfiltered spectra are quite noisy, and possible features (like a silicate $9.7 \mu \mathrm{m}$ absorption in PG $1501+106$ or a hot $4 \mu \mathrm{m}$ bump in PG 1718+481) in the Hanning smoothed plots have to be interpreted with caution. (The ISOCAMCVF spectra between 10 and $16 \mu \mathrm{m}$ do not show any PAH features.)

accompanied by PAH emission to an extent typical for starburst galaxies.
Therefore, we searched for PAH signatures in the MIR spectra of PG quasars taken with ISOPHOT-S and ISOCAM-CVF. The MIR spectra are dominated by the strong continuum of hot dust. Although the spectra show small bumps at some of the predicted positions (Fig. 6), the flux levels below $100 \mathrm{mJy}$ are too low to allow for a reliable detection. Thus, only upper limits for the PAH $7.7 \mu \mathrm{m}$ feature are derived (at the order of at least $10 \%$ of the continuum level). These upper limits, however, are still quite high, resulting in $L_{\mathrm{PAH} 7.7 \mu \mathrm{m} \text { peak }} / L_{\mathrm{FIR}}$ ratios between 0.1 and 1 (Table 4 ). This is clearly above the typical ratios of about $0.05-0.1$ we find for ULIRGs and normal spiral galaxies (using the PAH $7.7 \mu$ m peak fluxes and the FIR fluxes listed in Klaas et al. 2001).

In conclusion, the current high upper limits allow for PAH emission in PG quasars the strength of which is consistent to that of starburst galaxies. In order to find (via the PAHs) decisive arguments in favour of or against starbursts, more stringent detections or limits have to be provided, hence more sensitive MIR spectra with an accuracy of better than $1 \%$ are required.

\subsubsection{Constraints from the radio-FIR correlation}

In starburst galaxies the FIR and radio luminosities are proportional to each other over many orders of magnitude (Condon 1992). A natural explanation for this correlation is that the radio emission is mostly due to supernovae. Its rate is proportional to the massive star formation rate, and thereby also to the FIR luminosity, provided that much of the radiation from massive stars is absorbed and reradiated by dust.

Figure 7 (top) shows the relation between the FIR and radio luminosities for the PG quasars. Strikingly, the radioquiet quasars in our sample (50\%) lie close to the relation for starburst galaxies. The other half shows a clear radio excess, being obviously radio-intermediate or radio-loud. This radioloudness is attributed to AGN and jet activity. The radio loudness in our sample varies over six orders of magnitude, with no apparent correlation with the infrared or optical luminosity. Although the range of the radio luminosities is large, the Condon relation appears to present a lower limit to the radio flux. Such a limit could not be understood, if the radio emission arose exclusively from AGN jets, whose activity has no obvious relation to the cold dust component. The fact that the Condon relation forms a lower limit may be a fortuitous coincidence unrelated to star formation, though we cannot imagine a natural explanation for such a limit for AGN without starbursts. This suggests that in the low FIR luminosity range of about $10^{11} L_{\odot}$ starbursts of that power accompany the quasar phenomenon. However, for objects with high FIR luminosities above $10^{12} L_{\odot}$ the SEDs typically peak in the MIR so that the far-infrared luminosity could just be the Rayleigh-Jeans tail contribution of the warm AGN heated dust (as discussed in more detail in Sect. 4.2.2). In this case the actual starburst contribution to $L_{\mathrm{FIR}}$ may be only $10 \%$ and if we relate this FIR luminosity stemming from starbursts $\left(L_{\mathrm{SB}} \sim 0.1 \cdot L_{\mathrm{FIR}}\right)$ with the radio supernova luminosity, in Fig. 7 (top) the high $L_{\mathrm{FIR}}$ end would move down by a factor of about ten, leading effectively to a deviation from the Condon relation.

Next we probe the following assumptions: (1) If starburst activity were important in quasars, then one would expect those 

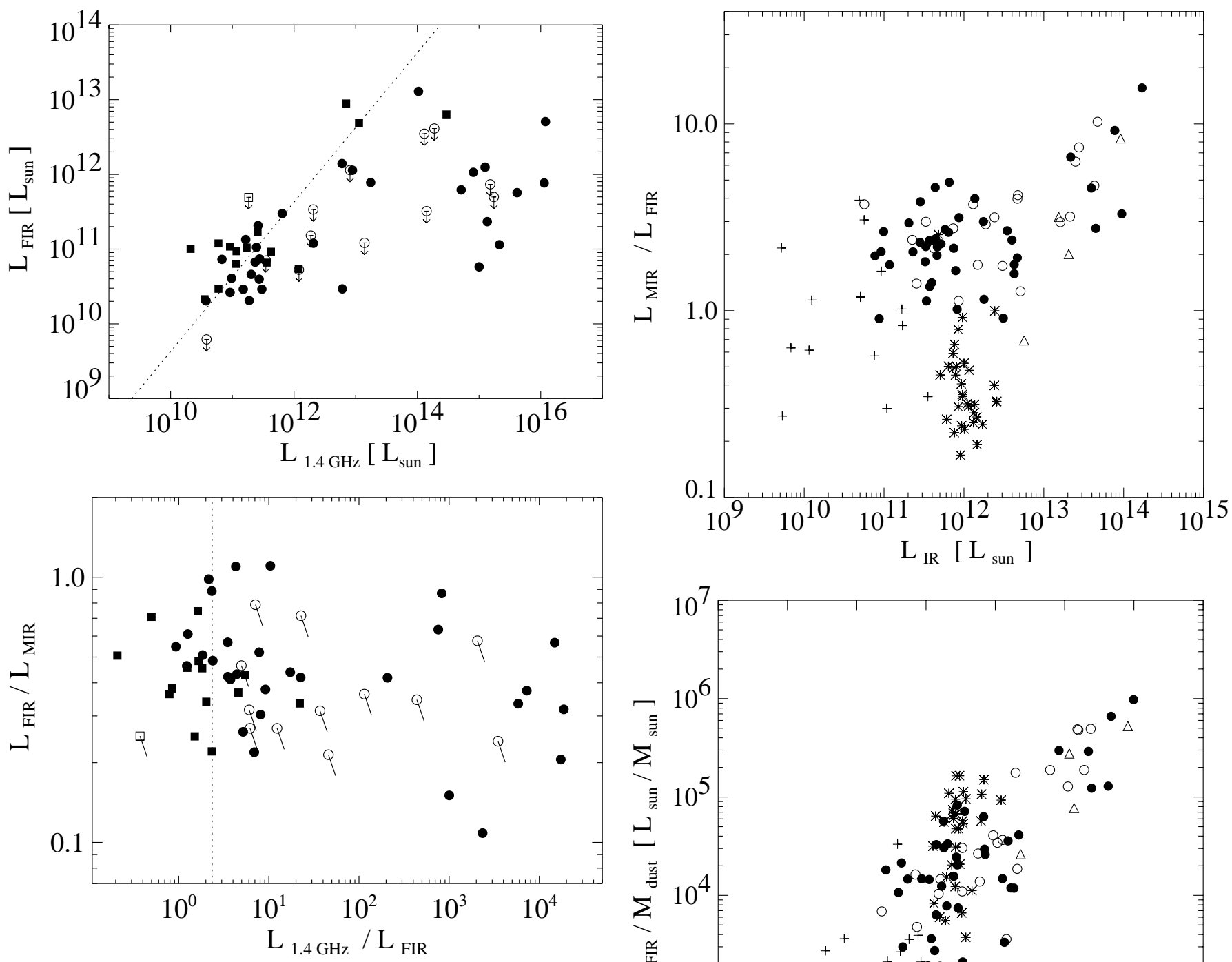

Fig. 7. Comparison of IR and Radio luminosities. Filled and open symbols refer to detections and upper limits, respectively. Circles and squares denote sources with $1.4 \mathrm{GHz}$ fluxes measured and extrapolated from $5 \mathrm{GHz}$, respectively. The dotted lines indicate the FIR-radio correlation for starburst galaxies from Condon (1992).

quasars with a high $L_{\mathrm{FIR}} / L_{\mathrm{MIR}}$ ratio (i.e. a relatively strong starburst component) to concentrate in a regime of the $L_{\text {radio }} / L_{\mathrm{FIR}}$ ratio similar to that of starburst galaxies. (2) If, on the other hand, in the radio-loud quasars with a high $L_{\text {radio }} / L_{\text {FIR }}$ ratio the AGN and jet activity traced a later state of evolution (with less starbursts), then they should show a lower $L_{\mathrm{FIR}} / L_{\mathrm{MIR}}$ ratio than the radio-quiet quasars (unless jet triggered starbursts become important). As a test for these possibilities, Fig. 7 (bottom) shows the distribution of $L_{\mathrm{FIR}} / L_{\mathrm{MIR}}$ versus $L_{\text {radio }} / L_{\mathrm{FIR}}$. The lack of any trend, however, does not allow to argue simply against the importance of starbursts, rather it means that one or both of the "if" conditions above are not fulfilled. Most likely the radio emission of radio-loud quasars is independent of the dust emission properties, since $L_{\text {radio }} / L_{\text {FIR }}$ is not correlated with $\alpha_{\mathrm{IR}}$ (Fig. 11 discussed in Sect. 4.3.2).

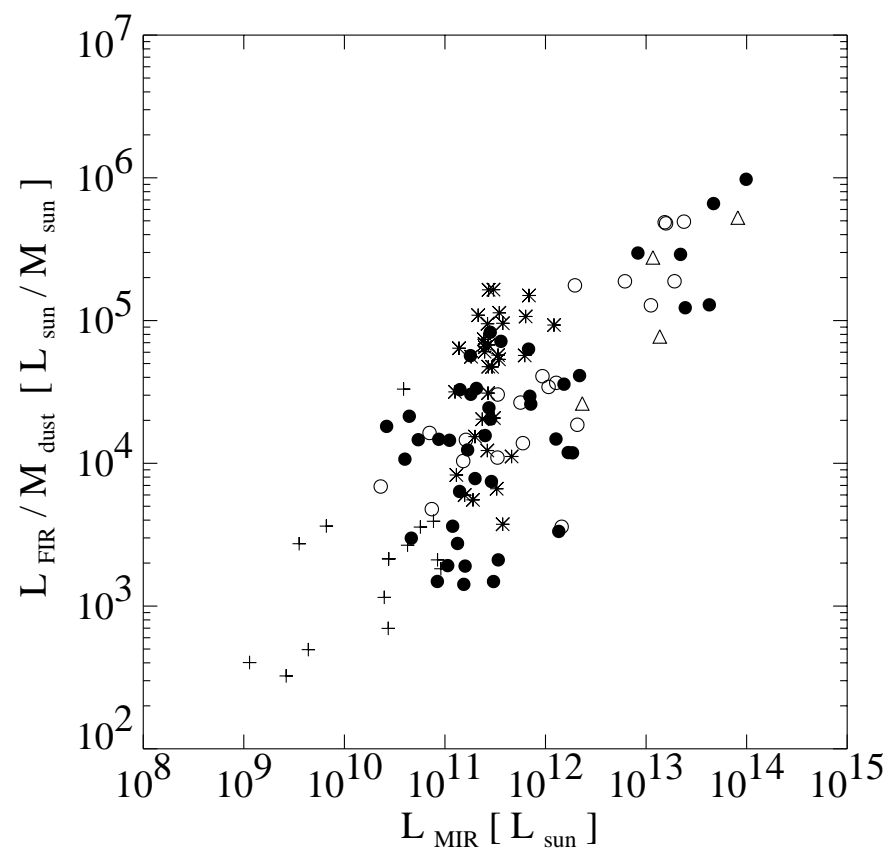

Fig. 8. Top: FIR/MIR luminosity ratio against IR luminosity. Bottom: FIR luminosity per dust mass against MIR luminosity. The symbols are: $\bullet=$ based on detections, $\circ=$ based on upper limits. For comparison also: $*=$ ULIRGs from Klaas et al. (2001), + = Seyfert 1 s from Spinoglio et al. (2002), $\Delta=$ HyLIGs from Verma et al. (2002, based on upper limits, and $M_{\text {dust }}$ is recalculated exactly as for the PG quasars).

\subsubsection{AGN dominance and limited starbursts in the hyperluminous $z=1 \mathrm{PG}$ quasars}

As shown in Fig. 8 (top) the hyperluminous sources have a higher MIR/FIR luminosity ratio than the bulk of the PG quasars. This ratio even is increased for the cases of FIR upper limits. For many hyperluminous sources $L_{\text {FIR }}$ reaches only one third or less of $L_{\mathrm{MIR}}$, suggesting that the AGN dominates even at FIR wavelengths. This issue was investigated for IR and optically selected hyperluminous IR galaxies by 
Rowan-Robinson (2000) on the basis of AGN torus and starburst models. Remarkably, the used models show little evidence for dominant starbursts in all hyperluminous quasars of Paper I. Assuming that the FIR luminosity traces the starburst power, the starbursts are at most ultraluminous, $L_{\mathrm{FIR}} \lesssim 10^{12} L_{\odot}$. This conservative estimate will be lower considering further arguments below.

In order to be independent of dust models, we proof the AGN dominance by a statistical comparison of our full quasar sample with properties of other galaxy classes:

Figure 8 shows also the nearby Seyfert 1s (from the $12 \mu \mathrm{m}$ AGN sample of Spinoglio et al. 2002), the nearby $(z<0.3)$ ULIRGs (from Klaas et al. 2001) and four IR selected hyperluminous IR galaxies (HyLIGs) at $z \approx 1$ (from Verma et al. 2002). Both classes, the Seyfert 1s and the ULIRGs, have (despite some scatter) on average a lower MIR/FIR luminosity ratio than the PG quasars, indicating a relatively low AGN and a high starburst contribution to the IR luminosity. The faintest one of the four HyLIGs actually seems to be similar to the nearby ULIRGs, but three HyLIGs lie close to the hyperluminous PG quasars. The PG quasars of more moderate luminosity lie just between the low luminosity Seyfert 1s and the hyperluminous quasars. This is consistent with the picture, that as a general trend the role of the AGN increases with total luminosity. This fact is well known for lower luminosity ranges (Fig. 4 in Miley et al. 1985; Fig. 8 in the review by Soifer et al. 1987), but here it is established observationally also for the hyperluminous range.

The trend of a $L_{\mathrm{MIR}} / L_{\mathrm{FIR}}$ ratio increase with total IR luminosity shows up also via the higher cut-off temperature of the thermal spectrum in the hyperluminous sources (Fig. 1, Table 2). For temperatures of 70-130 K, the far-infrared luminosity could just be the Rayleigh-Jeans tail of the warm dust, the emission of which peaks in the MIR. For example, the SED of a modified blackbody with emissivity $\propto \lambda^{-2}$ and $T=100 \mathrm{~K}$ peaks at $\lambda \approx 30 \mu \mathrm{m}$, resulting in $L_{\mathrm{FIR}} / L_{\mathrm{MIR}} \approx 0.1$. Even if a colder $T \approx 40 \mathrm{~K}$ dust component is present, the FIR+submm luminosity cannot raise significantly, say by at most $25 \%$, leaving $L_{\mathrm{FIR}} / L_{\mathrm{MIR}}$ clearly below 0.5 (Table 2 ). Hence, even if cold dust mass has escaped our detection, any starburst luminosity will be less than $\sim 10^{12} L_{\odot}$ estimated above.

Next, we consider the heating efficiency, i.e. the FIR luminosity normalised by dust mass. For a single blackbody $L_{\mathrm{FIR}} / M_{\text {dust }}$ is related to its temperature. But for a real mixture of several dust components the bulk of the dust mass is determined by the coldest component, while the bulk of the luminosity comes from a relatively small amount of the warm active dust (as long as it radiates in the FIR wavelength regime). Despite some possibly missed cold dust mass, the heating efficiency may provide clues to recognise AGN and starburst contributions: Certainly, for starbursts $L_{\mathrm{FIR}} / M_{\text {dust }}$ is expected to lie below a maximum threshold, since increasing the starburst power would require also more dust, i.e. interstellar matter out of which the stars are formed. On the other hand, energy production via a black hole is less restricted. The dust mass is comparable for all classes (Fig. 5), even if different dust properties are assumed as mentioned in Sect. 4.1. If the AGN had only little impact on the FIR luminosity in hyperluminous quasars, then $L_{\mathrm{FIR}} / M_{\text {dust }}$ should be similar to that of ULIRGs (and perhaps some less luminous quasars) where the FIR luminosity is starburst dominated. Figure 8 (bottom) shows the $L_{\mathrm{FIR}} / M_{\text {dust }}$ distribution against the MIR luminosity (as tracer for the AGN strength). The hyperluminous quasars clearly lie around and outside the high end of the distribution of all three comparison classes, (1) the Seyfert 1s, (2) the ULIRGs and (3) the luminous and ultraluminous PG quasars. This trend would be amplified, if the dust emissivity changed from $\beta=2$ to $\beta=1$ at the higher dust temperatures.

To conclude, the MIR/FIR luminosity ratio - and with some reservation also the $L_{\mathrm{FIR}} / M_{\text {dust }}$ distribution - argue in favour of a strongly dominating AGN contribution to the FIR emission of our $z=1$ hyperluminous quasars. For most of them the temperature of the cold end of the thermal spectrum seems to be higher than $70 \mathrm{~K}$. In these sources the starburst power is likely to be less than $10^{12} L_{\odot}$, i.e. it is clearly not hyperluminous.

\subsubsection{Progress of the AGN dust models}

The low optical extinction of the quasars indicates that the dust is distributed in a disk/torus geometry seen face-on or moderately inclined. The MIR and FIR as well as the blue luminosities are correlated over three to four orders of magnitude (Fig. 4). Since the total UV to optical luminosity is about ten times $L_{\mathrm{B}}$, we get $L_{\mathrm{UV}-\mathrm{opt}}>L_{\mathrm{IR}}$, hence the hot AGN core, in principle, can provide by far sufficient energy to heat all the dust. Also striking is the correlation between soft X-ray and FIR luminosity (Fig. 4, Table 3). But the problem is to find a scenario in which the central luminosity can efficiently be transported to reradiate from cooler outer regions. Most of the former AGN dust models (for example the torus study by Pier \& Krolik 1992, 1993), ran into difficulties, when trying to explain (1) the broad range of dust temperatures between about $1000 \mathrm{~K}$ and a few tens of Kelvin, and (2) a FIR/MIR luminosity ratio $\gtrsim 0.3$ found for many quasars (at $z<0.4$ ).

Recently, interesting progress has been achieved in modelling AGN heated IR SEDs: basically the models use a clumpy torus as proposed by Krolik \& Begelman (1988). All these findings complement each other in an ideal way for a pure AGN heating scenario:

Firstly, a promising physical model was presented by Zier \& Biermann (2001, 2002), where merging binary black holes create the dusty torus which, in addition, is patchy with gaps blown by strong stellar winds. Since a patchy torus tends to smear out after some time resulting in a homogeneous dust torus, a mechanism (namely stellar winds) is provided to explain, how to maintain the clumpiness.

Secondly, Nenkova et al. (2002) approximated a clumpy torus by a toroidal distribution of dense dusty clouds which are illuminated from one side by an AGN. Under reasonable assumptions their 1D-calculations (with the DUSTY code) yield SEDs with a broad temperature range, which also peak between 60 and $100 \mu \mathrm{m}$, similar to those observed (e.g. for 3C405 = Cyg A from Haas et al. 1998). Despite the simplification this investigation strongly suggests that starbursts are not required to provide the bulk of the FIR luminosity. 
Thirdly, Thiele \& Camenzind (2002) developed a 3D-code for calculating the radiative transfer for a proper dust model of silicates and graphites. They show that the temperature range can be broad even for exclusive AGN heating, if the total AGN power is not too high.

\subsubsection{Conclusions on the AGN-SB connection}

While in the past the FIR luminosity of quasars had challenged AGN models as well as the observational search for starburst tracers, now a somehow converse situation has been entered: the progress of the AGN dust torus models suggests that starbursts are not required as additional energy sources for the FIR emission of quasars, but the observations seem to provide more and more evidence for their presence in many quasars of our PG sample.

That the starburst power is on average related to the AGN power could be understood, if both were caused by the supply of gas during the process of galaxy merging. However, the strength of the starbursts relative to that of the AGN decreases with increasing total IR luminosity. While for some of our PG quasars at the lower end of the IR luminosity distribution the dust power of the starburst (measured as $L_{\mathrm{FIR}}$ ) may reach that of the AGN, our data leave no doubt, that, at least for the hyperluminous PG quasars at $z=1$, the AGN is the main power source for the dust emission also at FIR wavelengths. The physical reason for this trend could be that in our PG quasar sample

1. the starbursts can obviously reach only a FIR power of about $10^{10} \ldots 10^{12} L_{\odot}$, but they cannot exceed this value,

2. while the AGN powered IR emission is less restricted and can reach $10^{10} \ldots 10^{14} L_{\odot}$.

Therefore, and for the sake of lucidity, we adopt in the following discussion that starbursts play rather a minor role for powering the dust emission of the PG quasars.

\subsection{Evolution of the dust emission of quasars}

In the picture of AGN heated dust the IR emission depends on the power supplied by the AGN as well as on the amount and spatial distribution of the reprocessing dust around the illuminating AGN. In this section we consider the variety of the observed SED shapes, which turns out to be essentially inherent to the quasars, i.e. cannot entirely be explained by observational effects like inclination dependent extinction. We find that the SED shapes can be sorted so that they fit expectations about evolution of the quasars' dust emission, providing a natural understanding of the SED variety.

\subsubsection{The variety of optical and infrared SEDs}

Firstly, we consider some SED examples: Figs. 9 and 10 show the SEDs in $v F_{v}$ representation in which the optical to MIR wavelengths are best exposed. The $1 \mu \mathrm{m}$ dip between optical and IR wavelengths is clearly visible. We include here the optical wavelength range as a rough tracer for AGN properties. We fitted the optical and the near- to mid-infrared SEDs by

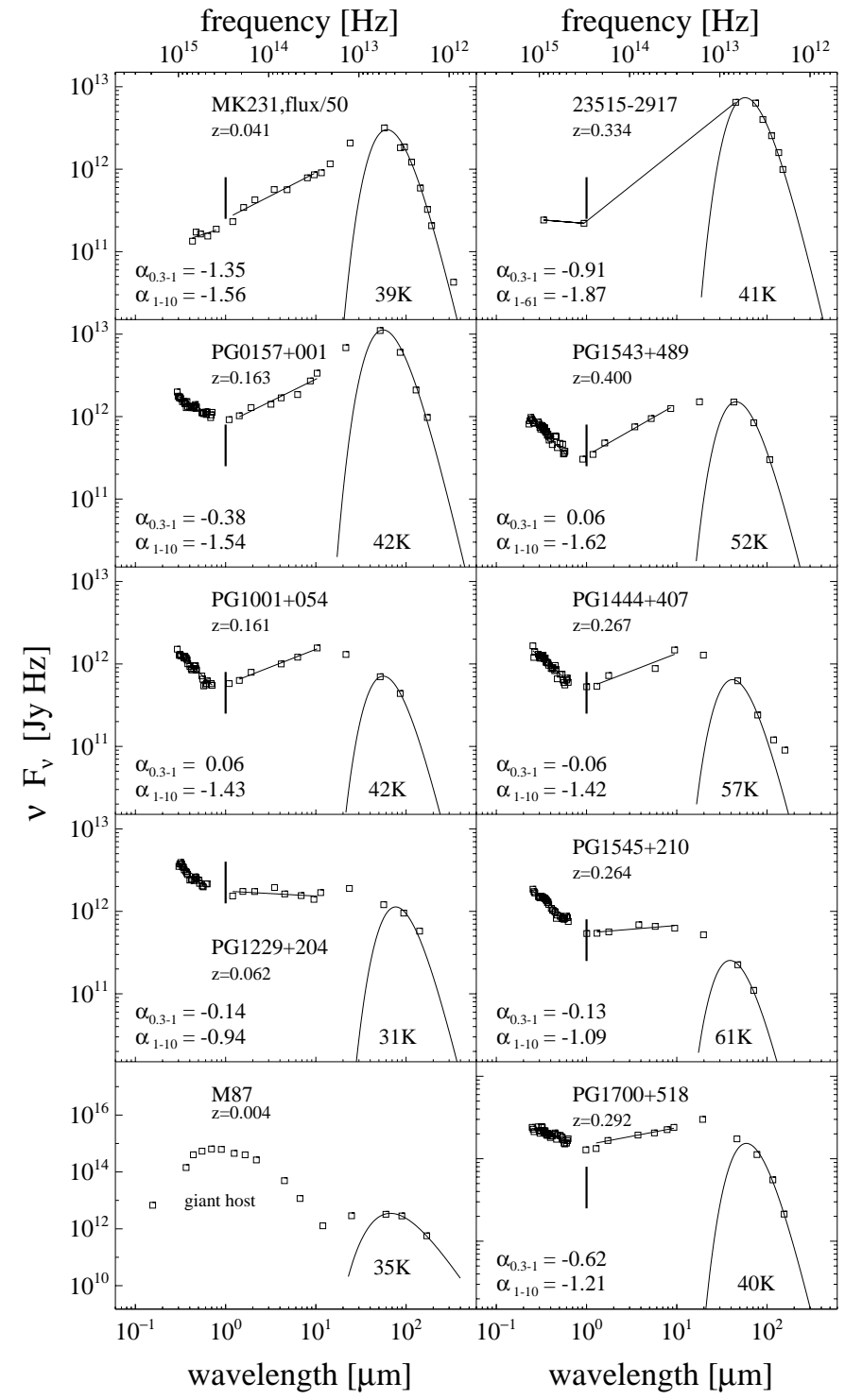

Fig. 9. Observed $v F_{v}$ for 10 selected quasars (frequency shifted to the quasar's restframe). The left and right columns contain sources at low and medium redshifts $(z<0.4)$, respectively. Data as in Fig. 1, upper limits are excluded, if not meaningful. The sources are sorted along the criteria proposed in Sect. 4.3.4: For comparison the first row contains two warm ULIRGs MK 231 and IRAS 23515-2917 (from Klaas et al. 2000). The IR slopes are steep for the FIR loud objects in the third row and then flatten in the fourth row. The fifth row shows the giant elliptical M 87 (for comparison from Haas et al.2003, in prep.). The optical slope is blue from the second to the fourth row, but is redder in the fifth row. The vertical bar indicates the position of the $1 \mu \mathrm{m}$ dip.

power-laws, as indicated by the lines. The SEDs exhibit a diversity which may be described by the following characteristics:

1. The spectral slope $\alpha_{\mathrm{IR}}=\alpha_{1-10 \mu \mathrm{m}}$ (Definition in Table 2). Examples for sources with steep slopes are PG 0157+001, PG 1543+210 and PG 1008+133 (at low, medium and high redshifts). Examples for sources with flat slopes are PG 1229+204, PG 1545+210 and PG 1718+481 (at low, medium and high redshifts of $z \approx 0.1,0.3$ and 1 respectively). 


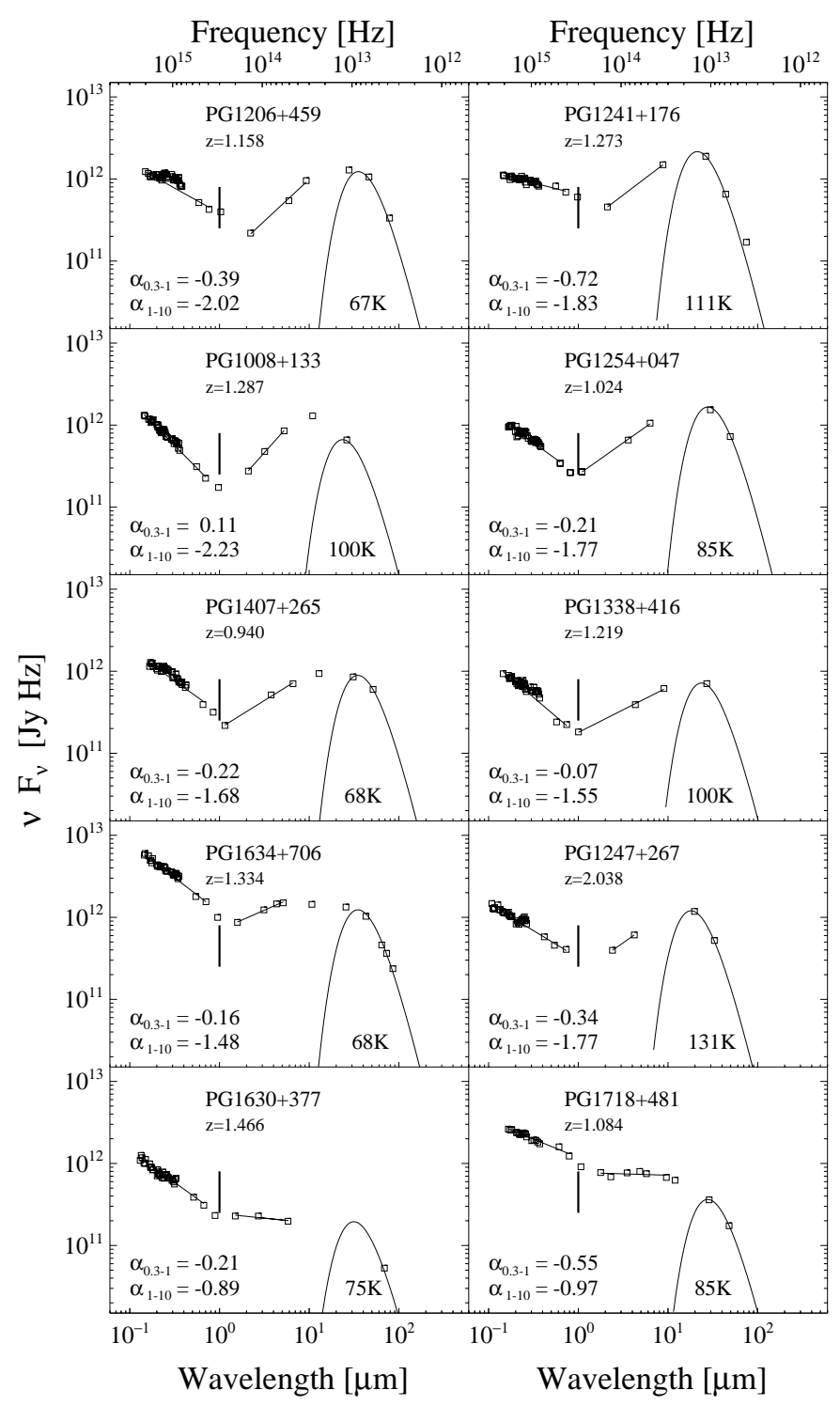

Fig. 10. Observed $v F_{v}$ for those 10 quasars of high redshift $z>0.9$ (frequency shifted to the quasar's restframe) for which sufficient optical and IR data are available. Data as in Fig. 1, upper limits are excluded, if not meaningful. The vertical bar at $1 \mu \mathrm{m}$ indicates where the dip is expected. This dip is shifted towards longer wavelengths for the quasars PG 1206+459 and PG 1241+176 (in row 1), both having also a slightly reddened optical slope. The other sources are sorted along IR slope being steep in rows 2, 3 and 4, and flat in row 5. The optical slope of these hyperluminous objects is generally blue in rows 2,3 and 4, but is redder for the objects in row 5; for details see Sect. 4.3.4.

2. The wavelength of the location of the $v F_{v}$ peak in the MIR at $\lambda \approx 10 \mu \mathrm{m}$ or FIR at $\lambda \gtrsim 40 \mu \mathrm{m}$. Examples for "MIR peakers" are PG 1001+054, PG 1444+407 and PG 1008+133 (at low, medium and high redshifts). Examples for " $F I R$ peakers" are PG0157+001, PG 1543+489 (peaking at $30-40 \mu \mathrm{m}$ ) and (to some extent) PG 1206+459 (at low, medium and high redshifts).

3. The temperature of the coldest dust component ranges from about $30 \mathrm{~K}$ in PG $1229+204$ to about $60 \mathrm{~K}$ in PG 1545+210 (at low and medium redshifts). At high redshifts $(1<z<2)$ it is less constrained (e.g. PG 1338+416), but seems to lie above $60 \mathrm{~K}$ (e.g. PG 1634+706, PG 1247+267). (In order to argue independently of the Planck function fit and more closely to the data, one could also use the drop of the SEDs at the cold side. Although the drop is not in all cases very sharp, the wavelength of this drop exhibits a considerable dispersion among our sample.)

4. The spectral slope $\alpha_{\text {opt }}=\alpha_{0.3-1 \mu \mathrm{m}}$ (Definition in Table 2). Examples for sources with blue slopes are PG 1001+054, PG 1545+210, and PG 1008+133 (at low, medium and high redshifts). Examples for sources with red slopes are PG 1404+226 (Table 2), PG 1700+518 and PG 1206+459 (at low, medium and high redshifts).

All possible combinations of these characteristics can be found: red (blue) optical and steep (flat) infrared SEDs, flat and steep SEDs which peak in the MIR, as well as flat and steep SEDs which peak in the FIR. A weak trend is that most of the FIR peakers are located a low redshift $(z<0.2)$. The sources at higher redshift are more luminous (Fig. 3), and the $L_{\mathrm{FIR}} / L_{\mathrm{MIR}}$ ratio decreases with luminosity, as mentioned in Sect. 4.2.2.

Furthermore, the few radio-loud sources in our sample do not show any preference for steep or flat slopes, etc. Examples can be seen in Fig. 1 and taken from Table 2: PG 1545+210 and PG 1704+608 have blue and red optical slopes, respectively; PG $1100+772$ has a quite flat IR slope, and PG 1718+481 has a red optical and flat IR slope. They are all at intermediate and high redshifts, and "MIR peakers", too. (PG 0007+106 and PG 1226+023 are not considered, since they have a quite flat radio spectrum, and PG $2308+098$ which has only a sparse optical filter coverage.)

\subsubsection{Search for statistical relations of $\alpha_{\mathrm{IR}}$}

Before we will consider physical explanations for the SED variety in the next sections, we will have a look at statistical properties of our sample. In the past the various IR data were noisier with sparse wavelength sampling which allowed only for crudely investigating $L_{\mathrm{MIR}}$ and $L_{\mathrm{FIR}}$. Now the ISO data provide templates of sufficient quality to allow, for the first time, the study of $\alpha_{\mathrm{IR}}$ for a statistically meaningful quasar sample. Figure 11 illustrates relations between the IR slope and other quantities:

1. Left column, top and bottom: The slopes $\alpha_{\mathrm{IR}}$ and $\alpha_{\mathrm{opt}}$ are randomly distributed, hence independent.

Similarly, the distribution of $\alpha_{\mathrm{IR}}$ and $\alpha_{\mathrm{X}}$ looks random. $\alpha_{\mathrm{X}}$ has been determined for a small PG subsample in common with ours from ASCA observations in the soft to hard $2-10 \mathrm{keV} X$-ray range by George et al. (2000). Note that $\alpha_{\mathrm{X}}$ does not show any trend with $\alpha_{\text {opt }}$. Neither does the spectral index from the optical to the soft X-ray regime $\alpha_{\text {ox }}$ (Laor et al. 1997) show any correlation with $\alpha_{\text {IR }}$ for their sample in common with ours. Hence, if central extinction inside the quasars dominated the variation of $\alpha_{\text {opt }}$, this should show up in $\alpha_{\mathrm{X}}$ or $\alpha_{\mathrm{oX}}$, too, contrary to what is observed.

2. Middle column, top: $\alpha_{\mathrm{IR}}$ shows a considerable dispersion, but also a marginal trend with the ratio of MIR-to-blue luminosity. A high MIR 10-40 $\mu$ m luminosity (with respect 

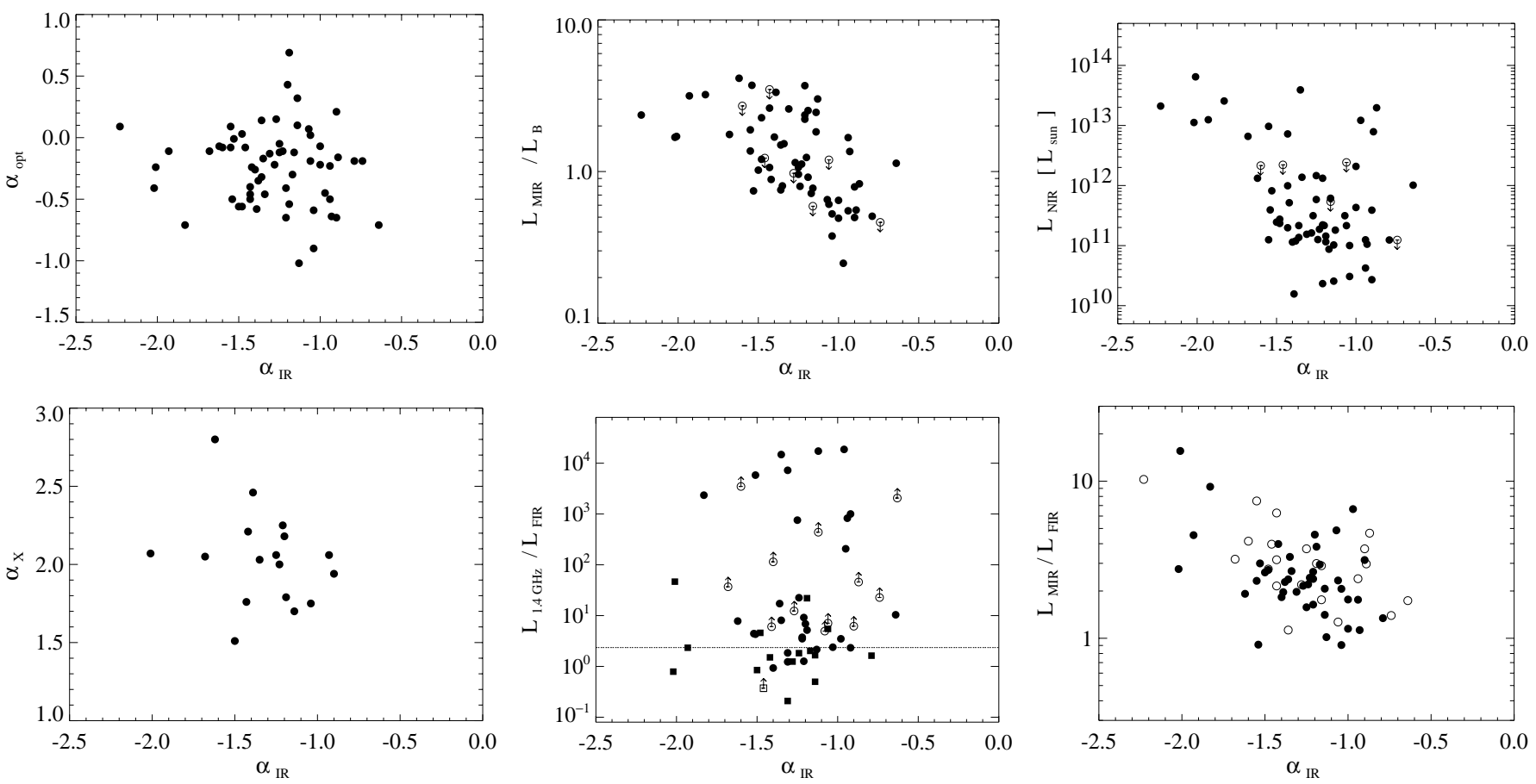

Fig. 11. Statistical relations of the spectral slope $\alpha_{\mathrm{IR}}=\alpha_{1-10 \mu \mathrm{m}}$ with other observables from Table 2 . The uncertainty in the slopes is about \pm 0.2 . $\left(\alpha_{\mathrm{opt}}=\alpha_{0.3-1 \mu \mathrm{m}}, \alpha_{\mathrm{X}}=\alpha_{2-10 \mathrm{kev}}\right.$.) The symbols are: $\bullet=$ based on detections, $\circ=$ based on upper limits (squares are as in Fig. 7).

to $L_{\mathrm{B}}$ ) requires, that between 1 and $10 \mu \mathrm{m}$ the IR flux grows, and this is exactly what is represented by $\alpha_{\mathrm{IR}}$. Therefore, the marginal correlation between $\alpha_{\mathrm{IR}}$ and $L_{\mathrm{MIR}} / L_{\mathrm{B}}$ is expected.

3. Middle column, bottom: $\alpha_{\mathrm{IR}}$ and the radio/FIR luminosity ratio are randomly distributed, hence independent. The width of the $\alpha_{\mathrm{IR}}$ distribution is similar for the radioloud and the radio-quiet quasars, the latter lying close to Condon's relation (dotted horizontal line).

4. Right column, top: For similar reasons as for (2), $\alpha_{\mathrm{IR}}$ also shows (despite a considerable dispersion) a marginal trend with the NIR luminosity. This trend just means: if there is much warm and hot dust showing up as high $L_{\mathrm{NIR}}$, then naturally the SED must grow from the $1 \mu \mathrm{m}$ dip up to the $10 \mu \mathrm{m}$ MIR wavelength range. The distribution, however, appears completely random, if we exclude the 5 hyperluminous sources with $L_{\mathrm{NIR}} \gtrsim 10^{13} L_{\odot}$ and $\alpha_{\mathrm{IR}}<-1.8$.

5. Right column, bottom: $L_{\mathrm{MIR}} / L_{\mathrm{FIR}}$ seems to be largly independent of $\alpha_{\mathrm{IR}}$. A marginal trend may be caused by the hyperluminous quasars, which lie towards the left and upper half of the diagram. Note that $L_{\mathrm{MIR}} / L_{\mathrm{FIR}}$ can also be interpreted as a kind of infrared spectral index, $\alpha_{\text {MFIR }}$ ranging between say 20 and $100 \mu \mathrm{m}$, but we maintain the terminology $L_{\mathrm{MIR}} / L_{\mathrm{FIR}}$, because between the MIR and FIR the emission does not generally follow a power-law, as is found for the NIR-to-MIR or optical wavelengths.

To conclude, the search for statistical trends between the spectral slope $\alpha_{\mathrm{IR}}$ and other quantities essentially confirmed the diversity and independence of the SED components, i.e. the hard and soft X-rays and the optical wavelengths tracing the AGN, the radio properties associated with a jet, and the near-, midand far-infrared emission tracing the dust around it.

\subsubsection{Possible reasons for the SED variety}

In this section we consider reddening of the optical AGN continuum and contributions from the host galaxy, and what can we learn from our IR data.

The optical photometry as presented by Neugebauer et al. (1987) includes correction for galactic foreground reddening according to Burstein \& Heiles (1982) as listed in Elvis et al. (1994). We have also considered the data as dereddened by Elvis et al. and, in addition, we performed a dereddening according to the most actual COBE/DIRBE data by Schlegel et al. (1998, with values listed in the NED) and the extinction curves by Cardelli et al. (1989). In general, the $A_{V}$ values are small $(<0.3)$ and occasional differences between the Burstein \& Heiles and the Schlegel et al. reddening are small, too. Consequently, the choice between foreground reddening values has only a minor impact on $\alpha_{\text {opt }}$, also if one considers the uncertainty due to fitting. For comparison, choosing an $A_{V}$ value which is erroneous by 0.1 results in a fitted slope $\alpha_{\text {opt }}$ modified by 0.1 . Thus the dereddening differences are within the uncertainties of the slopes of about 0.2. Therefore, we conclude that the variety of optical slopes (ranging between 0.6 and -1.0 ) cannot be attributed to an improper correction of galactic foreground reddening.

Stellar light from the host galaxies could affect the slopes and the correlations of Sect. 4.3.2. But this effect is probably small, in particular for high luminosities, as was already suggested by Neugebauer et al. (1987) on the basis of their random distributions in optical and NIR properties. Furthermore, on high resolution images of 15 nearby PG quasars Surace et al. (2001, their Table 3 ) find a $B$-, $I$ - and $H$-band nuclear luminosity fraction of $80 \pm 10 \%, 67 \pm 15 \%$, and $60 \pm 15 \%$ respectively. Assuming that the host galaxy is represented by a 
blackbody of $T=4000 \mathrm{~K}$, then even a host contribution of $30 \%$ at $1 \mu \mathrm{m}$ to the observed SEDs does not significantly change the SED shapes in the log-log plots of Figs. 9 and 10. If for the 12 sources of Surace, Sanders \& Evans in common with our sample, which are all low luminosity quasars, we use the non-nuclear fraction as a measure for the relative host contribution and subtract a $T=4000 \mathrm{~K}$ host from the observed SEDs, on average the optical and IR slopes can change by +0.23 and -0.1 , respectively. For some low luminosity quasars like PG $0838+770$ or PG $1415+451$, which show also a curvature in their optical spectra (being redder at longer wavelength), the $1 \mu \mathrm{m}$ dip becomes more pronounced after such a host subtraction. Nevertheless, the impact of the host galaxy turns out to be too small to explain the dispersion of the SED shapes as well as the wide distribution of $\alpha_{\mathrm{opt}}$ and, in particular, $\alpha_{\mathrm{IR}}$. Also, any shift of the $1 \mu \mathrm{m}$ dip due to the contribution of a cool host galaxy might be small, in particular for the hyperluminous hot quasars.

The diversity of $\alpha_{\text {opt }}$ has long been recognized (Neugebauer et al. 1987), and is still puzzling, together with the diversity of X-ray properties (e.g. Laor et al. 1997; George et al. 2000). Standard models predict $\alpha_{\text {opt }} \approx 0.3$ for the thermal optical/UV continuum of a centrally irradiated accretion disk (review by Koratkar \& Blaes 1999). Consequently, the observed range between 0.5 and -1.0 for $\alpha_{\text {opt }}$ may partly be explained by superEddington accretion rates, but also extinction of the central region could play a role. As reviewed e.g. by Mac Alpine (1985) the determination of reddening towards an AGN either from line ratios or from continuum properties is far from being trivial. Since the quasars were optically identified via their blue colours (UV excess), one would expect, if at all, only moderate intrinsic extinction $\left(A_{V} \lesssim 0.3\right)$. For most of the quasars such low extinction is indicated by the current observational status, unless blue light from the AGN is scattered into the line of sight (review by Koratkar \& Blaes 1999).

The shape of the optical and IR SEDs could be influenced by our viewing angle of the system. In the picture of the dust torus, an inclination of the torus axis with respect to the line of sight, so that the torus edge is grazed, will hide the hottest central regions. Then the NIR emission will be suppressed, resulting in

1. a shift of the $1 \mu \mathrm{m}$ dip towards longer wavelengths,

2. steepening of $\alpha_{\mathrm{IR}}$, and

3. $\alpha_{\mathrm{opt}}$ will be reddened, unless scattering plays a role.

However, only two of our quasars (PG 1206+459 and PG 1241+176, with observed $\alpha_{\text {opt }} \approx-0.4$ and -0.7 , respectively) show such signatures of reddening (Fig. 10). It could correspond to $A_{V} \approx 0.7 \ldots 1.0$, if the intrinsic slope is $\alpha_{\mathrm{opt}}=0.3$ (adopting $A_{V}=0.1$ corresponds to a change in $\alpha_{\text {opt }}$ by -0.1 ). Also PG $1718+481$ could be inclined, since it shows a quite red optical slope, and the $1 \mu \mathrm{m}$ dip is shifted, but $\alpha_{\mathrm{IR}}$ is flat. Noteworthy, these three sources all are hyperluminous quasars, where the IR emission is clearly dominated by the hot and warm dust. In this case the absorbing and emitting dust may be "coupled", i.e. the dust may be distributed in a relatively simple geometry like a dense torus with a sharp edge, so that (beside the optical reddening) the suppression of the hottest central regions may become observable in our near- to midinfrared spectra.

The majority of our quasars do not show such signatures of reddening. The lack of correlations (e.g. between $\alpha_{\text {opt }}$ and $\alpha_{\mathrm{IR}}$ ) in Fig. 11 argues against extinction by a simple dust torus seen inclined. If the dust geometry is less regular without any sharp torus edge, then the emission of the entire dust surrounding the AGN is manifold as may be indicated in the ISO data, and it is "decoupled" from the dust column along our line of sight towards the nuclear optical emission. In this case our IR spectra cannot exclude that the observed range of $\alpha$ opt is partly caused by dust extinction, with a possible minor contribution of scattered light. However, it is difficult to imagine, that dust extinction is the only reason for the variety of the optical slopes. Also concerning the variety of the IR slopes, the discussion here shows that it cannot simply be explained by extinction effects or host galaxy contributions. Rather we suggest that for most of our quasars the status of the central accretion disk mainly controls the optical emission, while the amount and distribution of the absorbing and reemitting dust may be more complex.

\subsubsection{Evolutionary sequence for PG quasars}

The variety of the optical and IR SEDs could just be the consequence of evolution among the PG quasars. In this picture the dust emission acts as a tracer for the evolutionary history of the quasars. The starting point of this scenario assumes that quasars are preceeded by a dusty ULIRG phase as proposed by Sanders et al. (1988a,b). If quasars contain dust, preferentially during their earliest phases, then this dust might not disappear at once, rather its signatures should tell us about dedicated stages during the quasars' evolution. While former IR data did not allow the recognition of definite detailed signatures (due to limited sensitivity, wavelength coverage or sample size), we suggest that the sensitive ISO data identifies such signatures for the first time in our large PG quasar sample. Therefore we worked out, what would be expected for the evolution of the dust distribution and emission, and compared it with the observations.

Firstly, we consider the physical processes acting on an initially irregular dust distribution. Dissipative cloud collisions and angular momentum constraints lead to the organisation of dust clouds into a torus/disk like configuration. On the other hand, injection of kinetic energy via supernova explosions or radiative pressure and jets from the AGN tend to increase the turbulence and to destroy any organised structures. As a net result, however, they will only delay the settling of the irregular dust distribution into the dusty torus/disk. The time scale for the whole sequence may roughly be estimated from the extent of the initial dust distribution (in the order of kpc) and the typical sound speed of clouds (in the order of $100 \mathrm{~km} \mathrm{~s}^{-1}$ ), leading to $10^{8}$ years, consistent with the time scale derived from merger simulations (e.g. Mihos \& Hernquist 1996; Naab \& Burkert 2001). Thus, the time scale of dedicated quasar phases (identified below) might be in the order of $10^{7}$ years.

With regard to the emission, the dust which is initially heated by starbursts will be powered more and more by the AGN, until the black hole $(\mathrm{BH})$ begins to starve. Since the 
PG quasars are practically not extinguished, for the discussion here we will not consider extinction effects due to the aspect angle, i.e. within the unified schemes we assume a nearly faceon view onto the quasar. During the evolution both the optical and the IR luminosity will grow and finally decline, although not necessarily simultaneously. The large luminosity dispersion in our flux limited sample could be just due to the existence of powerful "big" and less powerful "small" quasars, hence it does not permit an interpretation of the increase in luminosity as a pure consequence of evolution: an old big quasar and a small young quasar in an active phase could both show the same luminosity. Important is, that the relative contribution of the warm and cool dust components change during the evolution, and that this is expected to show up in the shapes of the observed SEDs.

We have arranged the expected dust geometries and SEDs for such an evolution in a graphical scheme of idealised observational classes as illustrated in Fig. 12, with their properties summarized in Table 5. These classes are

0. cool ULIRGs: The dust is distributed irregularly over the sphere, and cool FIR emission heated by starbursts is prominent. The AGN is not yet formed properly or it is still deeply embedded, $\alpha_{\text {opt }}$ and $\alpha_{\mathrm{IR}}$ are red and flat, respectively.

1. warm ULIRGs: Due to dissipative cloud collisions and guided by angular momentum balance, the dust concentrates in the central region. The AGN is already powerful, but still dust enshrouded, so that $\alpha_{\text {opt }}$ may become slightly bluer, but is still quite red. The contribution of warm dust increases resulting in a higher $L_{\mathrm{MIR}} / L_{\mathrm{FIR}}$ ratio and $\alpha_{\mathrm{IR}}$ steepens.

2. young quasars: The dust has settled into the torus/disk-like configuration. The pole-on view towards the AGN becomes less extinguished, so that $\alpha_{\text {opt }}$ gets blue. The $1 \mu \mathrm{m}$ dip becomes prominent. The IR slope is controlled by the covering angle and the density of the dust distribution. A decline of covering angle or density will make $\alpha_{\mathrm{IR}}$ flatter. Since we cannot decide which of both processes dominates, we sketched only the case of a declining covering angle in Fig. 12. Starbursts are becoming outshone by the AGN.

3. evolved quasars: The torus is stabilised and the dust density grows. The torus may still be patchy (case $a$ ), so that the AGN can heat the dust also at larger distances. $L_{\mathrm{MIR}} / L_{\mathrm{FIR}}$ may still increase further. $\alpha_{\mathrm{IR}}$ is steep (cases $a$ and $b$ ), but could also become flat, depending on the amount of dust illuminated by the AGN (case $c$ ). $\alpha_{\text {opt }}$ is clearly blue. This phase comprises the most luminous objects.

4. old quasars: The central black hole activity (feeding) declines, $\alpha_{\text {opt }}$ gets red again. The relative contribution of AGN heated warm dust declines, as does the $L_{\mathrm{MIR}} / L_{\mathrm{FIR}}$ ratio, and $\alpha_{\text {IR }}$ gets flat.

5. dead starved quasars: The central black hole does not get enough fuel to provide significant radiation in the hot optical-UV regime. The optical and NIR emission is dominated by the bright host galaxy, while the mid- and farinfrared emission reflects the cool dust residuals. The optical and IR spectra are not of power-law shape.
This sequence of classes is of course an idealised scheme, but it provides a natural understanding of the variety of the SED shapes. In order to facilitate the intuitive perception of this scheme, we have also included the ULIRGs as classes 0 and 1, although all our PG quasars belong to the classes 2 to 4 , which are stages in the evolutionary scheme identified here for the first time. Next we compare the scheme with the observations: in Figs. 9 and 10 we have arranged the observed SED shapes so, that from top to bottom they actually fit the above scheme. At low and intermediate redshifts (Fig. 9), the classes range from warm ULIRGs to old quasars. At $z \approx 1$ (Fig. 10) all of the examples might belong to evolved quasars (class 3 ), consistent with the fact that they are hyperluminous. The first row contains inclined ones with some signatures of extinction; the three middle rows of Fig. 10 contain evolved quasars of type $3 \mathrm{~b}$ (having $T_{\text {cold }} \gtrsim 70 \mathrm{~K}$ ), while the last row shows two quasars resembling type $3 \mathrm{c}$ (with some possible extinction for PG 1718+481). The proposed class assignments for all examples shown in Figs. 9 and 10 are listed in Table 5, and for the entire PG sample (where possible) they are listed in Table 2. In this evolutionary scheme, our PG quasars populate the classes 2 and 3 , and some even the late phase 4 , whereby we choose the terminology young - evolved - old with respect to the dust signatures. The radio galaxy M 87 (=3C 274, IR SED from Haas et al. in prep.), which we assign class 5 , illustrates further a "dead quasar" with a starved black hole with a faint jet, and giant elliptical host and a faint dust emission.

The interpretation of the SED classes as part of an evolutionary sequence provides a new issue and it will have to be investigated, how far every quasar of class 2 needs to evolve along all the steps of the sequence. Nevertheless, going the other way round and considering a quasar of a given SED class, a quasar of say class $3 \mathrm{~b}$ does not exist ad hoc, rather when asking "what was the precursor of this object type?", a plausible candidate is one like class $3 \mathrm{a}$ in the proposed scheme. Moreover, in the picture of quasars as merger remnants, the amount and distribution of dust could also depend on the parent galaxies or distortions of an evolved quasar by a second merger event with a dust-rich galaxy, leading to deviations from simple straight forward expectations like in the proposed scheme. Larger data samples in the future may find further clues to this topic.

In principle, stellar light from bright host galaxies could also affect the SED shapes. As discussed in Sect. 4.3.3 host effects could be present to some extent in the optical wavelength range, but it is negligible in the IR range, in particular longwards of $5 \mu \mathrm{m}$. Furthermore, it is worth to note that in the IR guided sequence shown in Fig. 12, as well as indicated in the observational data in Figs. 9 and 10, the optical slope appears red at the beginning (class 0 and 1), then becomes blue during the phase of a clear view towards the AGN (class 2 and 3), and finally seems to become redder again - at least for some quasars in class 4 . Whether this IR guided sequence reflects also a sequence of AGN/black hole activity is beyond the scope of this paper and is left to future studies. (We do not consider, how big the BHs are at the beginning; just worth mentioning is that the typical growth rate of the $\mathrm{BH}$ mass with time, $M(t)=M_{\mathrm{o}} \times \mathrm{e}^{t / t_{\text {edd }}}$ with initial BH mass $M_{\mathrm{o}} \sim 10^{7} M_{\odot}$ 


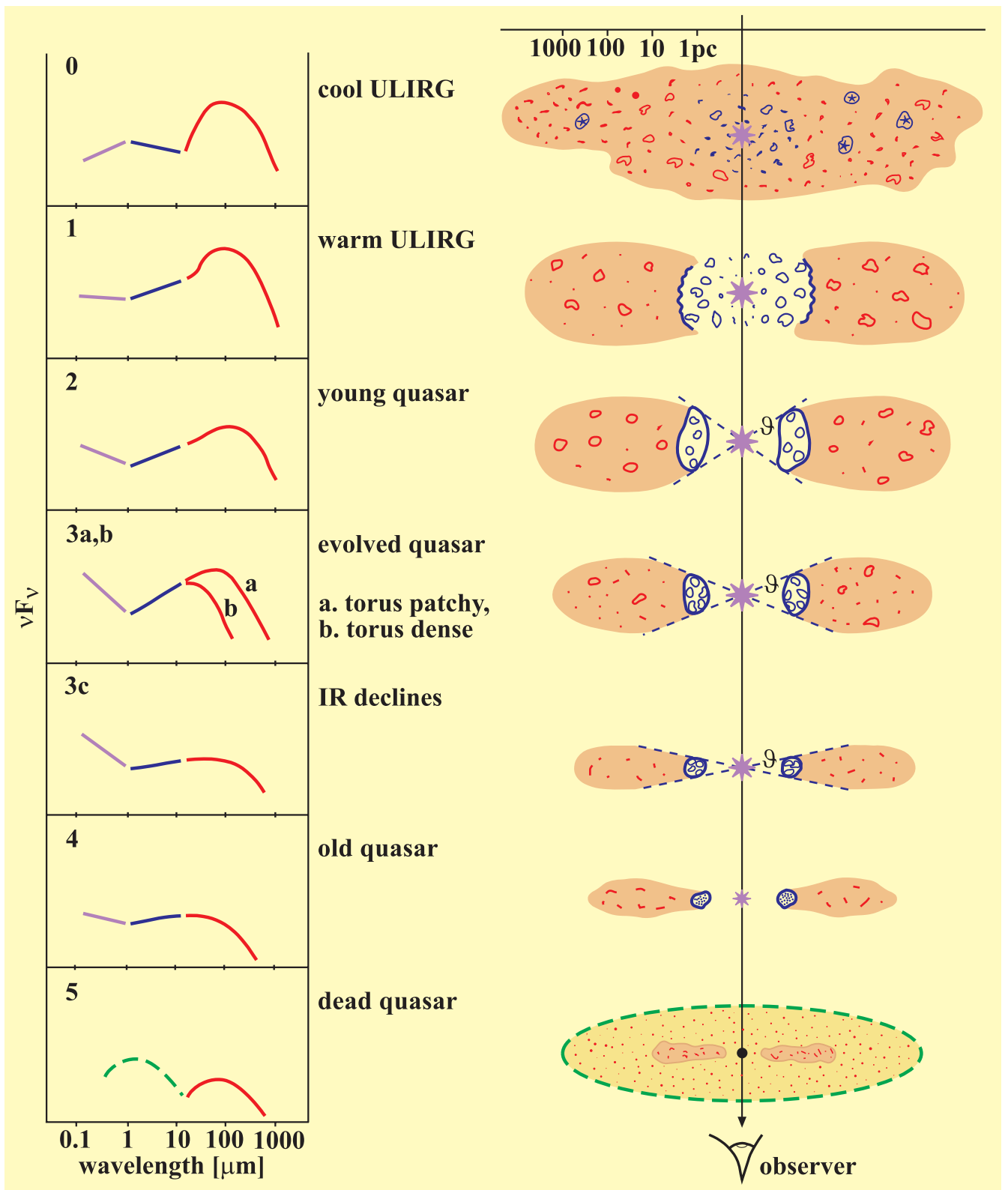

Fig. 12. Scheme of quasar evolution for the dust distribution surrounding the AGN, with corresponding SEDs. During the evolution (from top to bottom) the dust distribution rearranges, settling more and more into a torus/disk like configuration. The corresponding SEDs show an initial FIR bump, then an increase in MIR emission and a steepening of the infrared slope, both of which finally also decrease. The AGN strength grows, then stays high and finally declines, as is marked by the size of the $\star$ and shows up in the SEDs by the optical slope.

and Eddington time $t_{\text {edd }}=4 \times 10^{7}$ years, in principle, would provide a quasar-like $\mathrm{BH}$ of $10^{8} M_{\odot}$ during $10^{8}$ years without difficulty.)

So far the classification of the SED shapes was based on visual inspection, guided by the features FIR bump, MIR bump, steepness of the NMIR slope and - to a lesser degree - also the value of $T_{\text {cold }}$ and the steepness of the optical slope. It is desirable to provide also quantitative measures for the sorting procedure. As an attempt for such an approach, Fig. 13 shows the distribution of our classes in the parameter space of $L_{\mathrm{MIR}} / L_{\mathrm{FIR}}, \alpha_{\mathrm{IR}}$ and $\alpha_{\mathrm{opt}}$. The classes, in fact, concentrate on different regions in the three diagrams, although they are not clearly disjoint. For example in the diagram $L_{\mathrm{MIR}} / L_{\mathrm{FIR}}$ versus $\alpha_{\mathrm{IR}}$ : class $3 \mathrm{a}$ lies in the middle of the diagram, class $3 \mathrm{~b}$ extends towards the upper left, while class $3 \mathrm{c}$ lies more toward the righthand side. In view of the complexity of the objects, it would be overdone to expect a clear separation of the classes with only three parameters. One reason for the overlaps may be that during the anticipated evolution the parameters move "up" and then "down", for example $L_{\mathrm{MIR}} / L_{\mathrm{FIR}}$ grows and finally declines. Nevertheless, the trends found in the diagrams (Fig. 13) indicate that our eyeball SED classifications basically match these formal quantities. Future studies may be able to refine the classification, perhaps considering also the total luminosity (which is yet indefinite as mentioned above). As a vision, they may find evolutionary tracks for quasars in such diagrams in analogy to those for the pre-main-sequence stellar birthlines and the giants' branches in the Hertzsprung-Russel diagram. 
Table 5. Scheme of optical and infrared parameters during the quasar evolution shown in Fig. 12. The examples refer to Figs. 9 and 10.

\begin{tabular}{|c|c|c|c|c|c|c|c|c|}
\hline class & $\begin{array}{l}0 \\
\text { ULIRG } \\
\text { cool }\end{array}$ & $\begin{array}{l}1 \\
\text { ULIRG } \\
\text { warm }\end{array}$ & $\begin{array}{l}2 \\
\text { QSO } \\
\text { young }\end{array}$ & $\begin{array}{l}3 \mathrm{a}, \mathrm{b} \\
\text { QSO } \\
\text { evolved }\end{array}$ & inclined & $\begin{array}{l}3 \mathrm{c} \\
\text { QSO } \\
\text { evolved-old }\end{array}$ & $\begin{array}{l}4 \\
\text { QSO } \\
\text { old }\end{array}$ & $\begin{array}{l}5 \\
\text { QSO / radio galaxy } \\
\text { dead, BH starved }\end{array}$ \\
\hline$\alpha_{\mathrm{opt}}$ & red & medium & blue & blue & & blue & medium & red host galaxy \\
\hline$\alpha_{\mathrm{IR}}$ & flat & steep & steep & medium & & flat & flat & dto \\
\hline $1 \mu \mathrm{m} \operatorname{dip}$ & not seen & shallow & medium & deep & $>2 \mu \mathrm{m}$ & medium & shallow & - \\
\hline covering angle & sphere & large & medium & medium/low & & medium/low & low & - \\
\hline$L_{\mathrm{FIR}} / L_{\mathrm{MIR}}$ & high & medium & medium & low & & low & medium & medium/high \\
\hline$T$ (cold) $[\mathrm{K}]$ & $20-40$ & $20-40$ & $30-50$ & $\geq 50$ & & $\gtrsim 30$ & 230 & $20-40$ \\
\hline \multicolumn{9}{|l|}{ examples } \\
\hline low $z(\approx 0.1)$ & $17208-0014^{*}$ & MK 231* & PG 0157+001 & PG $1001+054$ & & PG 1229+204 & & M 87 \\
\hline medium $z(\approx 0.3)$ & & $23515-2917^{*}$ & PG $1543+489^{x}$ & PG 1444+407 & & PG $1545+210$ & PG $1700+518$ & \\
\hline \multirow[t]{6}{*}{$\operatorname{high} z(\approx 1)$} & & & & PG $1008+133$ & PG $1206+459$ & PG 1630+377 & & \\
\hline & & & & PG $1247+267$ & PG $1241+176$ & PG $1718+481^{+}$ & & \\
\hline & & & & PG $1254+047$ & & & & \\
\hline & & & & PG $1338+416$ & & & & \\
\hline & & & & PG $1407+265$ & & & & \\
\hline & & & & PG 1634+706 & & & & \\
\hline
\end{tabular}

\footnotetext{
* From Klaas et al. (2001).

${ }^{x}$ Could also belong to class 3 a.

${ }^{+} \alpha_{\text {opt }}$ appears red and $1 \mu \mathrm{m}$ dip shallow, but if it is shifted towards $2 \mu \mathrm{m}$, then PG 1718+481 could also be inclined.
}

The coarse difference between the SEDs of ULIRGs and quasars, combined with the high bolometric luminosities, led Sanders et al. (1988a,b) to propose the general evolutionary link between these two galaxy types. The diversity of the quasar IR SEDs was already noticed for IRAS samples with sparse wavelength coverage and limited sensitivity (e.g. Barvainis 1990; Elvis et al. 1994) or for the small ISO sample of 17 sources in Paper I, but without allowing stringent interpretations like evolution among PG quasars. The sensitive ISO data of a sufficiently large sample of 64 PG quasars allow for the first time to establish the details of the SED variety and to classify them. Combining now the SED diversity with the idea of sorting along evolutionary expectations, provides the main new result here, which may stimulate future studies.

To conclude, the observed variety of SEDs can be associated with and sorted into physically meaningful classes, which reflect the amount and distribution of the reprocessing dust around the AGN. These classes can naturally be understood as a consequence of evolution of the quasars' dust distribution and heating.

\subsubsection{Trends of IR emission with host morphology?}

If quasars are merger remnants in a post-ULIRG phase, then one would expect that - compared with older relaxed and more evolved quasars - the younger quasars are those which show both (1) more ULIRG-like cool dust and (2) more distortions in the (optical or NIR) morphology of their host galaxy.

In order to test this prediction, we collected host galaxy information for 34 of our 64 PG quasars in the literature. Table 2 lists a compilation of several data sets compiled by Clements (2000) and observed by Surace et al. (2001). As in Clements (2000), we distinguish simply between distorted and undistorted hosts. Figure 14 shows the distribution of the $L_{\mathrm{FIR}} / L_{\mathrm{MIR}}$ ratio and the FIR luminosity for these 34 PG quasars. They are indistinguishable for those with and without host distortions, respectively. Also, the distributions of $\alpha_{\mathrm{IR}}$ or the histograms of SED classes $(2, \ldots 4)$ look indistinguishable for disturbed and regular hosts. Furthermore, no trend of predominant spiral or elliptical hosts with the IR properties can be found. Notably, we cannot confirm the claim by Clements (2000), that those PG quasars, which are luminous in the IRAS $60 \mu \mathrm{m}$ band, are more distorted than the faint ones, a result which might be an artefact produced by the numerous IRAS upper limits. Also, the youth of a quasar may be better identified by the $L_{\mathrm{FIR}} / L_{\mathrm{MIR}}$ ratio than by the absolute $60 \mu \mathrm{m}$ luminosity.

Our result, however, does not conflict with the evolutionary scheme proposed in Fig. 12, since (1) any cold dust clouds and starbursts of the warm ULIRG phase may be predominantly confined to the nuclear region, hence are very compact and not resolvable even on HST images, and (2) any extended starbursts, which are not deeply dust enshrouded and could show up also via a knotty optical/NIR morphology, likely play only a minor role in the PG quasars. An evolutionary trend of a declining IR emission with relaxed host morphology may exist among the PG quasars, but the available data do not yet allow this to be established.

\subsection{Hyperluminous quasars and cosmic evolution}

Here we will not discuss cosmological effects within our PG quasar sample, although it is striking that - as already known from many optical investigations - at low redshift $(z<0.4)$ no hyperluminous quasars are present (Fig. 3). Different redshift bins of our flux limited PG sample contain sources of different luminosities, hence the bins probably do not contain representatives of comparable luminosity or type which are seen just at different cosmic epochs. Also, any physical evolution from a hyperluminous $z=1$ quasar into a less luminous one at $z<0.4$ would just be speculative.

Of special interest, however, is to compare sources of similar luminosity, but from different cosmic epochs. Very high 

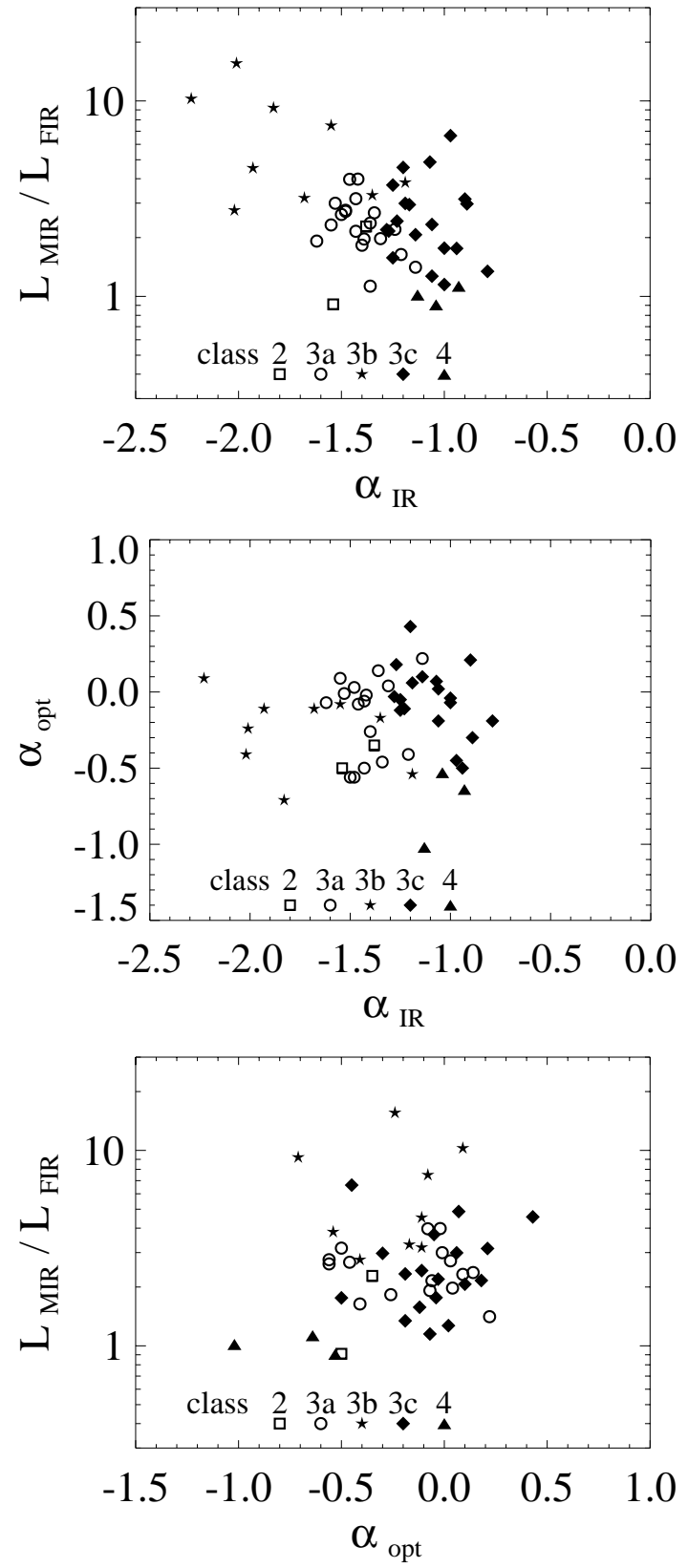

Fig. 13. Locus of the SED classes in the $L_{\mathrm{MIR}} / L_{\mathrm{FIR}}-\alpha_{\mathrm{IR}}-\alpha_{\mathrm{opt}}$ parameter space. Although the SED classes were visually determined, they concentrate at distinct regions in the three diagrams. (The data are as in Fig. 11.)

redshift $(z=4)$ quasars have been discovered in the Digitized Palomar Sky Survey (PSS quasar catalog, e.g. Kennefick et al. 1995) and from the Sloan Digital Sky Survey (SDSS quasar samples, e.g. Fan et al. 2000). These quasars have $M_{\mathrm{B}}$ estimates between -26 and $-29 \mathrm{mag}$, comparable to our hyperluminous PG quasars at $z=1$ with $M_{\mathrm{B}} \lesssim-27$ mag and $L_{\mathrm{IR}} \gtrsim 10^{13} L_{\odot}$. Due to the K-correction the $z=4$ quasars are sufficiently bright to be detected also in (sub-)millimetre surveys. In this section we compare our $z=1$ PG quasars with $z=4$ quasars from the PSS and SDSS, in order to study the influence of cosmic epoch on the amount of dust and its heating sources.

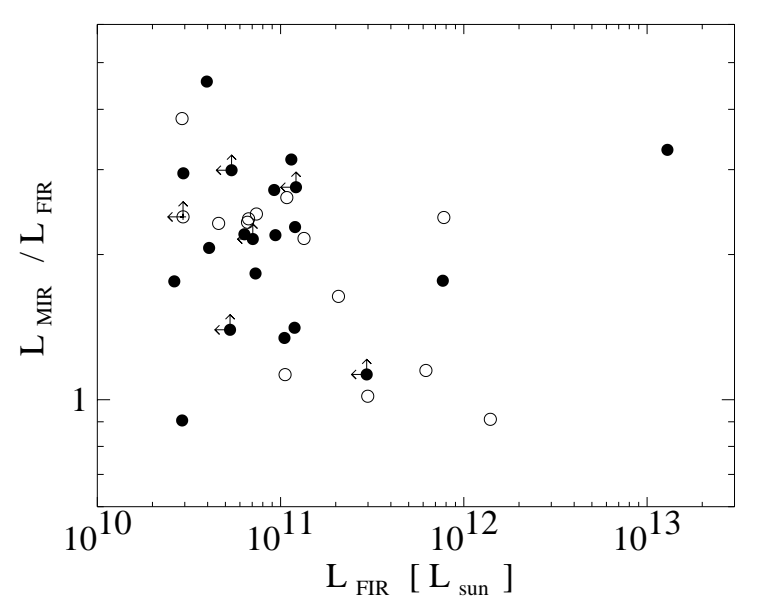

Fig. 14. $L_{\mathrm{MIR}} / L_{\mathrm{FIR}}$ ratio versus $L_{\mathrm{FIR}}$ for $\mathrm{PG}$ quasars with undisturbed $(\bullet)$ and disturbed (०) host morphology. The arrows indicate upper limits for $L_{\mathrm{FIR}}$.

\subsubsection{Observational differences}

For such a comparison between our "local" $z=1$ hyperluminous quasars and those at very high redshifts, the influence of two observational differences has to be checked:

Firstly, the optical selection of the local PG quasars is based on the intrinsic UV-blue continuum, while the $z=4$ quasars are identified via the Lyman break using two-colour diagrams. Actually, the two-colour criteria seem to be so strict, that only sources with a strong $\mathrm{Ly}_{\alpha}$ line and low extinction are selected. Nevertheless, while the PG quasars definitely contain objects with a blue continuum, the high $z$ quasar samples could, in principle, contain also objects, which exhibit a red intrinsic continuum, hence would be different from the PG quasars. Such a difference, however, is not supported by the current observational status of the $z=4$ quasars: The mean restframe UV slope of the SDSS $z=4$ quasars lies in the range around -0.67 and -0.79 (Fan et al. 2000, 2001), and the mean restframe optical slope lies around -0.5 (determined from $J, H, K$ photometry by Pentericci et al. 2002, in preparation, private communication by H.W. Rix \& L. Pentericci). These values are similar to that of the PG sample (mean $\alpha_{\text {opt }} \approx-0.5$, Fig. 11). Therefore, with respect to the $z=1 \mathrm{PG}$ quasars, we do not expect a bias in favor of or against cool dust emission from (presumably reddened) hyperluminous starbursts in the PSS and SDSS $z=4$ quasars.

Secondly, the part of the rest frame dust emission, which is well constrained, differs: for the local sources it is the mid- and far-infrared range (i.e. the warm side of the dust emission) and for the distant ones it is the (sub-)millimetre range, i.e. the cold end of the thermal spectrum. While the warm side provides conclusions about the AGN-starburst relation from energy considerations (like the MIR/FIR luminosity ratio adopting it as indicator for $L_{\mathrm{AGN}} / L_{\mathrm{SB}}$ ), the cold side provides constraints from temperature and mass of the coldest dust in these sources. 


\subsubsection{On hyperluminous starbursts in $z=4$ quasars}

Several millimetre and sub-millimetre surveys with MAMBO and SCUBA detected dust emission from about $30 \%$ of the PSS and SDSS $z=4$ quasars observed (e.g. Omont et al. 2001; Carilli et al. 2001a,b; Bertoldi \& Cox 2002). The FIR to mm luminosity of the $z=4$ quasars is suggested to be starburst driven, and not AGN-powered. Remarkably, the inferred starbursts are hyperluminous $\left(L_{\mathrm{FIR}-\mathrm{mm}} \gtrsim 10^{13} L_{\odot}\right)$. Before we accept these hyperluminous starbursts for the cosmological comparison (in Sect. 4.4.3), we address some caveats:

1. Although the PSS and SDSS quasars contain a huge amount of dust, the central AGN of these quasars is very little extinguished with typical $A_{V}<0.3$ (Fan et al. 2001). The optical selection from a total population of very luminous dusty objects could pick out just those "exotic" objects, which - perhaps by chance - provide optical paths of low extinction towards the center. Since it is hard to believe that a chaotic dusty system can provide such a path, the dust distribution should follow a disk-like geometry seen face-on. However, hyperluminous starbursts - in particular if they are produced by a merging galaxy group - may be expected to disturb any somehow ordered disklike dust distribution. This complication could be lessened, if the dusty starbursts were more moderate.

2. The conclusions about the starburst dominance in the $z=4$ quasars are essentially based on the dust temperature of about $50 \mathrm{~K}$ adopted for most of the sources. This average temperature is determined only for few sources with several measured SED points, e.g. by Benford et al. (1999) and Priddey \& Mc Mahon (2001). It could be biased towards rather low values, since it is derived exclusively from those sources detected at $\mathrm{mm}$ wavelengths, while warmer $(T \gtrsim 70 \mathrm{~K})$ sources of a given MIR-FIR luminosity would provide mainly upper limits at $1.2 \mathrm{~mm}$ (Fig. 2 in Omont et al. 2001), hence no definite temperature estimate. Actually, dust temperatures of about $80 \mathrm{~K}$ have been found for some $z \approx 4$ quasars (Chini \& Krügel 1994), and $T \approx 90 \mathrm{~K}$ for IRAS F10214+4724, the lensed $z=2.2$ AGN-SB HyLIG (Manning \& Spinrad 2001). This temperature range is more similar to that of hyperluminous PG quasars at $z=1$. One of our PG quasars (PG 1247+267) at $z=2$ has the highest temperature of more than $120 \mathrm{~K}$ (Fig. 10), whereby the data allow for an additional cooler $(T \lesssim 70 \mathrm{~K})$, but by far less luminous dust component. Therefore the hyperluminous $z=4$ quasars likely exhibit a range of dust temperatures between say 50 and $120 \mathrm{~K}$, certainly not unexpected in view of the variety of dust emission properties discussed in Sect. 4.3.

3. The fit of the FIR to mm SEDs by a modified blackbody is not unique. Instead of a single component with $T=50 \mathrm{~K}$ and emissivity index $\beta=1.5$ used for the $z=4$ quasars, two temperature components of emissivity index $\beta=2$ could be a better physical description of the dust content in complex interacting galaxy systems, as was shown by Klaas et al. (2001) for the detailed IR-to-mm SEDs of nearby ULIRGs. The current SED sampling of the $z=4$ quasars is too sparse to establish such a two component decomposition, but the one component fit yielding an "effective" $\beta=1.5$ already indicates that two $\beta=2$ components could be present, say a warm hyperluminous
AGN dominated one of $T \approx 70 \mathrm{~K}$ and a clearly less luminous cool starburst component of $T \approx 30 \mathrm{~K}$.

4. Some $z=4$ quasars show also huge $\mathrm{H}_{2}$ gas masses $\left(\sim 10^{11} M_{\odot}\right)$ derived from CO detections (e.g. Cox et al. 2002; Carilli et al. 2002). The gas-to-dust mass ratio appears consistent with that for nearby galaxies and ULIRGs, supporting the correctness of the dust mass estimates. However, the determination of a high dust mass consistent with the $\mathrm{H}_{2}$ gas mass is not unique; it could be achieved via the adopted dust temperature of $50 \mathrm{~K}$ using $\beta=1.5$, or two $\beta=2$ dust components as mentioned above.

5. The use of a single blackbody with $T=50 \mathrm{~K}$ leads to a hyperluminous object with $L_{\mathrm{FIR}-\mathrm{mm}}$ of some $10^{13} L_{\odot}$ which is completely ascribed to starbursts. The derived radio/FIR luminosity ratio seems to be consistent with that of starburst galaxies (e.g. Carilli et al. 2001b). However, the MIR luminosity is not known, so that any Rayleigh-Jeans-tail contribution from an AGN dust component to the FIR-mm luminosity is ignored. Here, a decomposition into a two temperature component model could provide a more realistic picture.

6. Some of the caveats on hyperluminous starbursts inferred from a $50 \mathrm{~K}$ dust component could be alleviated, if the $z=4$ quasars were gravitationally lensed, certainly not an unexpected possibility in view of the huge distance. For example, a $z=4$ quasar lensed by a factor of about 5 would intrinsically be more similar to the normal luminous to ultraluminous PG quasars which typically show a cold thermal end of about $50 \mathrm{~K}$. However, for a few examined cases the evidence for lensing is low, and in view of the high detection rate of about $30 \%$ the probability that most of the (sub-)millimetre detected quasars are strongly lens-amplified is low, too. Further clues to these puzzles have to be left to future investigations.

To conclude, about $30 \%$ of the $z=4$ PSS and SDSS quasars observed show (sub-)millimetre signatures which could be due to hyperluminous starbursts. However, these signatures have to be considered with caution. The main uncertainty lies in the dust temperature and the unknown AGN contribution to the FIR luminosity.

\subsubsection{AGN-SB difference with cosmic epoch}

The comparison of hyperluminous quasars from the PG sample at $z=1$ with those from PSS and SDSS at very high redshift $(z=4)$ yields:

The selection criteria for the $z=4$ PSS and SDSS quasars and their restframe UV slopes measured so far (compared with those of the PG quasars) do not suggest a bias in favor of or against dust emission from hyperluminous starbursts.

At early cosmic epochs $(z=4)$ quasars appear accompanied by starbursts which in some cases may reach hyperluminous strength. At later epoch $(z=1)$ in the local universe the starbursts which accompany quasars obviously do not reach that high power and are clearly outshone by the AGN.

If the low and high incidence of hyperluminous starbursts at $z=1$ and $z=4$, respectively, can be further established, then the reason for this difference could be a much denser early universe with stronger and more frequent merger events. This 
supports the hypothesis of coeval formation of massive black holes and galaxy spheroids via merging galaxies at high redshifts, in order to explain the relation between black hole mass and velocity dispersion of the galaxy bulge (e.g. Gebhardt et al. 2000).

\section{Summary}

The IR to mm SEDs of 64 PG quasars obtained with ISO, about half of which were supplemented by MAMBO and SCUBA, represent excellent templates providing evidence for luminous to hyperluminous dust emission in quasars. The power-law shape and the smoothness of the IR spectra suggest that the central AGN is the main heating source, and that starbursts play only a minor role, even at FIR wavelengths. The SEDs show a diversity which we suggest to trace the physical evolution of the quasars' dust distribution and heating, and a classification of the SEDs as well as an evolutionary scheme is proposed. Signatures of cosmic evolution are identified for the hyperluminous quasars at $z=1$, which do not show the hyperluminous starbursts suggested to exist in (sub-)millimetre detected quasars in the denser early universe at $z=4$.

Acknowledgements. We wish to thank the anonymous referee for constructive suggestions. The ISOPHOT Data Centre at MPIA is supported by Deutsches Zentrum für Luft- und Raumfahrt e.V. (DLR) with funds of Bundesministerium für Bildung und Forschung, grant No. 50 QI 0201. IRAM is supported by INSU/CNRS (France), MPG (Germany) and IGN (Spain). This research is essentially based on the Data Archives of ISO, SCUBA, ROSAT and the NVSS. For literature and photometry search NED and SIMBAD were used. It is a pleasure to thank IRAM for discretionary observing time with the $30-\mathrm{m}$ telescope at Pico Veleta.

\section{References}

Barvainis, R. 1990, ApJ, 353, 419

Barvainis, R., Alloin, D., \& Antonucci, R. 1989, ApJ, 337, L69

Barvainis, R., Alloin, D., Guilloteau, S., \& Antonucci, R. 1998, ApJ, 492, L13

Bechtold, J., Elvis, M., Fiore, F., et al. 1994, AJ, 108, 374

Benford, D. J., Cox, P., Omont, A., Phillips, T. G., \& McMahon, R. G. 1999, ApJ, 518, L65

Bertoldi, F., \& Cox, P. 2002, A\&A, 384, L11

Burstein, D., \& Heiles, C. 1982, AJ, 87, 1165

Cardelli, J. A., Clayton, G. C., \& Mathis, J. S. 1989, ApJ, 345, 245

Carilli, C. L., Bertoldi, F., Omont, A., et al. 2001a, ApJ, 555, 625

Carilli, C. L., Bertoldi, F., Rupen, M. P., et al. 2001b, AJ, 122, 1679

Carilli, C. L., Cox, P., Bertoldi, F., et al. 2002, ApJ, 575, 145

Cesarsky, C., Abergel, A., Agnese, P., et al. 1996, A\&A, 315, L32

Cesarsky, D., \& Blommaert, J. 2001, ISOCAM calibration accuracies (ESA publications)

Chini, R., Kreysa, E., \& Biermann, P. L. 1989a, A\&A, 219, 87

Chini, R., Biermann, P. L., Kreysa, E., \& Gemünd, H.-P. 1989b, A\&A, 221, L3

Chini, R., \& Krügel, E. 1994, A\&A, 288, L33

Clements, D. L. 2000, MNRAS, 311, 833

Condon, J. J. 1992, ARA\&A, 30, 575

Cox, P., Omont, A., Djorgovski, S. G., et al. 2002, A\&A, 387, 406

de Vaucouleurs, G., de Vaucouleurs, A., Corwin, Jr. H. G., et al. 1991, Third reference catalog of bright galaxies, Version 3.9 (Berlin: Springer-Verlag)
Elvis, M., Wilkes, B. J., McDowell, J. C., et al. 1994, ApJS, 95, 1

Engels, D., Hagen, H.-J., Cordis, L., et al. 1998, A\&AS, 128, 507

Evans, A. S., Frayer, D. T., Surace, J. A., \& Sanders, D. B. 2001, AJ, 121,3286

Falcke, H., Bower, G. C., Lobanov, A. P., et al. 1999, ApJ, 514, L17

Fan, X., Strauss, M. A., Schneider, D. P., et al. 2000, AJ, 119, 1

Fan, X., Strauss, M. A., Schneider, D. P., et al. 2001, AJ, 121, 54

Gallagher, S. C., Brandt, W. N., Laor, A., et al. 2001, ApJ, 546, 795

Gebhardt, K., Bender, R., Bower, G., et al. 2000, ApJ, 539, L13

George, I. M., Turner, T. J., Yaqoob, T., et al. 2000, ApJ, 531, 52

Haas, M., Chini, R., Meisenheimer, K., et al. 1998, ApJ, 503, L109

Haas, M., Müller, S. A. H., Chini, R., et al. 2000, A\&A, 354, 453 (Paper I)

Haas, M., Klaas, U., \& Bianchi, S. 2002, A\&A, 385, L23

Hagen, H.-J., Engels, D., \& Reimers, D. 1999, A\&AS, 134, 483

Hildebrand, R. H. 1983, QJRAS, 24, 267

Kellermann, K. I., Sramek, R., Schmidt, M., et al. 1989, AJ, 98, 1195

Kennefick, J. D., de Carvalho, R. R., Djorgovski, S. G., et al. 1995, AJ, 110, 78

Kessler, M. F., Steinz, J. A., Anderegg, M. E., et al. 1996, A\&A, 315, L27

Kessler, M. F., Müller, T. G., Arviset, C., García-Lario, P., \& Prusti, T. 2000, The ISO Handbook vol. 1, SAI/2000-035/Dc (ESA publ.)

Klaas, U., Laureijs, R., Radovich, M., Schulz, B., \& Wilke, K. 2002, ISOPHOT calibration accuracies, V5.0 (adapted to OLP10) ISO Explanatory Library Doc. SAI/1998-092/Dc (ESA publications)

Klaas, U., Haas, M., Müller, S. A. H., et al. 2001, A\&A, 379, 823

Koratkar, A., \& Blaes, O. 1999, PASP, 111, 1

Kreysa, E., Gemünd, H.-P., Gromke, J., et al. 1998, SPIE, 3357, 319

Krolik, J. H., \& Begelman, M. C. 1988, ApJ, 329, 702

Laor, A., Fiore, F., Elvis, M., Wilkes, B. J., \& McDowell, J. C. 1997, ApJ, 477, 93

Laureijs, R., \& Klaas, U. 1999, ISOPHOT Error Budgets V1.0, ISO Explanatory Library Doc. SAI/98-091/dc (ESA publications)

Laureijs, R., Klaas, U., Richards, P. J., Schulz, B., \& Abraham, P. 2002, The ISO Handbook, vol. IV: PHT - the Imaging PhotoPolarimeter, Version 2.0, SAI/99-069/dc (ESA publications)

Léger, A., \& Puget, J.-L. 1989, ARA\&A, 27, 161

Lemke, D., Klaas, U., Abolins, et al. 1996, A\&A, 315, L64

Manning, C., \& Spinrad, H. 2001, AJ, 122, 113

McAlpine, G. M. 1985, in Astrophysics of active galaxies and quasi-stellar objects, ed. J. S. Miller (University Science Books), 259

Meisenheimer, K., Haas, M., Müller, S. A. H., et al. 2001, A\&A, 372, 719

Mihos, J. C., \& Hernquist, L. 1996, ApJ, 464, 641

Miley, G., Neugebauer, G., \& Soifer, B. T. 1985, ApJ, 293, L11

Naab, T., \& Burkert, A. 2001, in The Central Kiloparsec of Starbursts and AGN, ed. J. H. Knapen, J. E. Beckman, I. Shlosman, \& T. J. Mahoney, ASP Conf. Proc., 249, 735

Nenkova, M., Ivezić, Z., \& Elitzur, M. 2002, ApJ, 570, L9

Neugebauer, G., Miley, G. K., Soifer, B. T., \& Clegg, P. E. 1986, ApJ, 308,815

Neugebauer, G., Green, R. F., Matthews, K., et al. 1987, ApJS, 63, 615

Omont, A., Cox, P., Bertoldi, F., et al. 2001, A\&A, 374, 371

Oyabu, S., Kawara, K., Tsuzuki, Y., et al. 2001, A\&A, 365, 409

Pier, E. A., \& Krolik, J. H. 1992, ApJ, 401, 99

Pier, E. A., \& Krolik, J. H. 1993, ApJ, 418, 673

Polletta, M., \& Courvoisier, Th. 1999, A\&A, 350, 765

Polletta, M., Courvoisier, Th., Hooper, E. J., \& Wilkes, B. J. 2000 A\&A, 362, 75

Priddey, R. S., \& McMahon, R. G. 2001, MNRAS, 324, L17 
Rowan-Robinson, M. 1995, MNRAS, 272, 737

Rowan-Robinson, M. 2000, MNRAS, 316, 885

Sanders, D. B., Soifer, B. T., Elias, J. H., et al. 1988a, ApJ, 325, 74

Sanders, D. B., Soifer, B. T., Elias, J. H., et al. 1988b, ApJ, 328, L35

Sanders, D. B., Phinney, E. S., Neugebauer, G., et al. 1989, ApJ, 347, 29

Schlegel, D. J., Finkbeiner, D. P., \& Davis, M. 1998, ApJ, 500, 525

Schmidt, M., \& Green, R. F. 1983, ApJ, 269, 352

Soifer, B. T., Houck, J. R., \& Neugebauer, G. 1987, ARA\&A, 25, 187

Solomon, P. M., Downes, D., Radford, S. J. E., \& Barrett, J. W. 1997, ApJ, 478, 144

Spinoglio, L., Andreani, P., \& Malkan, M. A. 2002, ApJ, 572, 105

Stickel, M., Lemke, D., Klaas, U., et al. 2000, A\&A, 359, 865

Surace, J. A., Sanders, D. B., \& Evans, A. S. 2001, AJ, 122, 2791

Thiele, M., \& Camenzind, M. 2003, A\&A, submitted
Véron-Cetty, M. P., \& Véron, P. 1998, ESO Scientific Report: A Catalogue of Quasars and Active Nuclei 8th ed. (Garching: ESO) Verma, A., Rowan-Robinson, M., McMahon, R., \& Efstathiou, A. 2002, MNRAS, 335, 574

Wang, T. G., Brinkmann, W., Yuan, W., et al. 2000, ApJ, 545, 77

Wilkes, B. J., Tananbaum, H., Worrall, D. M., et al. 1994, ApJS, 92, 53

Wilkes, B. J., Hooper, E. J., Mc Leod, K., et al. 1999, ESA-SP, 427, 845

Wilkes, B. J., Hooper, E. J., Mc Leod, K., et al. 2000, in ISO Surveys of a Dusty Universe, ed. D. Lemke, M. Stickel, \& K. Wilke, Lecture Notes in Physics, 548, 177

Yuan, W., Siebert, J., \& Brinkmann, W. 1998, A\&A, 334, 498

Zier, C., \& Biermann, P. L. 2001, A\&A, 337, 27

Zier, C., \& Biermann, P. L. 2002, A\&A, 396, 91 